\title{
THE ERROR IN SPATIAL TRUNCATION FOR SYSTEMS OF PARABOLIC CONSERVATION LAWS
}

\author{
HUNG-JU KUO
}

ABSTRACT. In this paper we investigate the behavior of the solution of

$$
\begin{gathered}
u_{t}=D u_{x x}-f(u)_{x}, \\
u(0, x)=u_{0}(x) \in L^{\infty}, \quad u(t, \pm L)=u^{ \pm},
\end{gathered}
$$

where $t \geq 0$ and $x \in[-L, L]$. Solutions of this equation are considered to be approximations to the solutions of the corresponding parabolic conservation laws. We obtain decay results on the norms of the difference between the solution for $L$ infinite and the solution when $L$ is finite.

\section{INTRODUCTION}

This paper is concerned with the solution of certain parabolic systems of the form

$$
u_{t}+f(u)_{x}=D u_{x x}, \quad(x, t) \in \mathbf{R} \times \mathbf{R}^{+},
$$

with nonsmooth initial data

$$
u(x, 0)=u_{0}(x) \in L^{\infty}
$$

and boundary values

$$
u( \pm L, t)=u^{ \pm}, \quad t>0,
$$

where $u=\left(u_{1}, \ldots, u_{m}\right), f$ is a smooth vector function of class $C^{2}$ which may not be defined for all values of $u$, and $D$ is a positive diagonal matrix. These systems are conservation laws with dissipation and arise in a variety of applications including chemical chromatography and the flow of compressible fluids and gases. We apply our results to these examples in $\S 4$.

In [11], Nishida and Smoller designed an explicit finite difference scheme under consideration here and showed that the approximate solutions do in fact converge to a unique, classical solution for isentropic gas dynamics in Lagrangian coordinates. Hoff and Smoller [7] applied the same scheme to the general system (1.1)-(1.2) for $D=\mu I$ and established a precise error bound for their numerical scheme when the initial data has finite total variation. Both of these works depend crucially on the existence of an invariant region for the

Received by the editors February 13, 1987.

1980 Mathematics Subject Classification (1985 Revision). Primary 35K45; Secondary 65M15. 
system (1.1)-(1.2) with $D=\mu I$ in which the matrix $f^{\prime}$ is bounded; solutions are obtained as the limit of solutions of an explicit finite difference scheme possessing the same invariant region, under the assumption that the initial data has finite total variation. The disadvantage of their scheme is that it requires the usual parabolic stability condition $\Delta t / \Delta x^{2} \leq$ const and therefore is of limited usefulness. Using implicit schemes, one may expect to improve the mesh condition from a parabolic one to a linear one where $\Delta t / \Delta x \leq$ const. However, a computable implicit scheme cannot be applied to an infinite spatial domain. The purpose of our work is then to estimate the convergence rates for the solution of the boundary value problem to the solution of the Cauchy problem. Therefore, one can design more flexible numerical schemes for the boundary value problem instead of the Cauchy problem and use the result as the approximate solution for the Cauchy problem (see Kuo [9]).

There are no assumptions made about the smoothness and the values at infinity of the initial data. Thus, one can even consider oscillatory data or "Riemann problem" data.

In the present paper, we establish a precise result (Theorem 3.10) by obtaining the following convergence rates: For a given $j>0, L>0$, and $0<t \leq T$,

$$
\begin{aligned}
& \left\|u(\cdot, t)-u^{L}(\cdot, t)\right\|_{\infty,[-L, L]} \\
& \quad \leq c(j, T)\left[P\left(u_{0}, u^{ \pm}\right)( \pm L)+\frac{1}{L^{j}}\left\|u_{0}-u^{ \pm}\right\|_{\infty}+\left\|u_{0}\right\|_{\infty} \exp \left(\frac{L^{2}}{4 \mu t}\right)\right]
\end{aligned}
$$

where $u$ and $u^{L}$ and the solutions of (1.1)-(1.2) and (1.1)-(1.3) respectively, and $P\left(u_{0}, u^{ \pm}\right)$represents a polynomial-like decay rate in the real number $L$, and $\mu=\|D\|_{\infty}$.

Because our goal is to establish the effect on the solution of this spatial truncation, both the Cauchy problem (1.1)-(1.2) (in which $u_{0} \in L^{\infty}(\mathbf{R})$ ) and the boundary value problem (1.1)-(1.3) (in which $u_{0} \in L^{\infty}([-L, L])$ ) will be discussed in the present paper. In $\S 2$, we shall prove the existence of a global smooth solution $u$ of class $C^{2}$ for (1.1)-(1.3). Then, we will derive, in detail, the rigorous convergence rate (1.4) in $\S 3$. Also, some applications are given in $\S 4$.

Finally, we let $D=\left(\mu_{1}, \ldots, \mu_{m}\right), K_{i}$ be the usual heat kernel

$$
K_{i}(x, t)=\frac{1}{\sqrt{4 \pi \mu_{i} t}} \exp \left(\frac{x^{2}}{4 \mu_{i} t}\right),
$$

and $G_{l}^{L}(x, y, t)$ be the Green's function for the heat equation with Dirichlet boundary conditions on $[-L, L] \times \mathbf{R}^{+}$:

$$
G_{i}^{L}(x, y, t)=\sum_{j=-\infty}^{\infty} K_{i}(x-4 j L-y, t)-K_{i}(4 j L+2 L-x-y, t) .
$$


The solution $u(t)=u(\cdot, t)$ of $(1.1)-(1.2)$ then satisfies the standard representation

$$
u(t)=K(t) * u_{0}-\int_{0}^{t} K_{x}(t-s) * f(u(s)) d s,
$$

where $K(t)=\left(K_{1}(t), \ldots, K_{m}(t)\right)^{T}$, and the solution $u^{L}(t)=u^{L}(\cdot, t)$ of $(1.1)-$ (1.3) then satisfies

$$
u^{L}(t)=G^{L} *_{B}\left(u_{0}-h^{L}\right)+h^{L}-\int_{0}^{t} G_{y}^{L}(t-s) *_{B} f\left(u^{L}(s)\right) d s
$$

where

$$
h^{L}(x)=\frac{x+L}{2 L} u^{+}-\frac{x-L}{2 L} u^{-},
$$

$G^{L}(t)=\left(G_{1}^{L}(t), \ldots, G_{m}^{L}(t)\right)^{T}$, and $*_{B}$ denotes

$$
G^{L}(t) *_{B} u_{0}(x)=\int_{-L}^{L} G^{L}(x, y, t) u_{0}(y) d y .
$$

It is well known that

$$
\left\|G^{L}(t)\right\|_{\infty} \leq \frac{C}{t} \text { and }\left\|D_{x}^{p} D_{y}^{q} G^{L}(t)\right\|_{1} \leq C_{p+q} t^{-(p+q) / 2},
$$

where $p \geq 0, q \geq 0$, and $C_{0}=1$ (see Ladyzhenskaya [10]).

This research represents part of a dissertation submitted to the Graduate School of Indiana University in candidacy for the degree of Doctor of Philosophy. The author extends a most hearty thanks to Professor D. Hoff for his constant encouragement and numerous helpful suggestions.

\section{THE EXISTENCE AND SMOOTHNESS OF GLOBAL SOLUTIONS}

We shall prove the existence and uniqueness of global solutions in the sense of $\S 1$ for (1.1)-(1.3) which have the implicit representation form (1.8). Local existence of solutions can be established by a simple iteration argument and by using Schauder estimates for the derivatives of solutions. However, for most examples of interest, the flux is not defined for all values of $u$. To prove global existence, it is therefore necessary to bound the sup norm of the solution in such a way that $u$ remains in an invariant set $S^{\prime}$ in which $f$ is defined and Lipschitz continuous.

Most of the inequalities which we will encounter have a certain form. For later use, we therefore derive the following lemma which gives a Gronwall-type inequality.

Lemma 2.1. Let $A(t), B(t)$, and $g(t)$ be positive.

(a) Suppose that $A(t)$ is continuous and $g(t)$ is is integrable on $[\delta, T]$ for some $\delta \in(0, T]$, and assume that

$$
|g(t)| \leq A(t)+M \int_{\delta}^{t} \frac{1}{\sqrt{t-s}}|g(s)| d s \quad \forall t \in[\delta, T]
$$


for some $M \geq 0$. Then we have

$$
|g(t)| \leq A(t)+M \int_{\delta}^{t} \frac{1}{\sqrt{t-s}} A(s) d s+C(t, \delta) \int_{\delta}^{t} A(s) d s
$$

a.e. on $[\delta, T]$, where

$$
C(t, \delta)=\pi M^{2} \exp \left(\pi M^{2}(t-\delta)\right)(1+2 M \sqrt{t-\delta}) .
$$

(b) Suppose that $B(t)$ is continuous, integrable on $(0, T]$ and $g(t)$ is integrable on $(0, T]$, and assume that

$$
|g(t)| \leq B(t)+M \int_{0}^{t} \frac{1}{\sqrt{t-s}}|g(s)| d s \quad \forall t \in(0, T]
$$

for some $M \geq 0$. Then we have

$$
|g(t)| \leq B(t)+M \int_{0}^{t} \frac{1}{\sqrt{t-s}} B(s) d s+C(t) \int_{0}^{t} B(s) d s
$$

a.e. on $(0, T]$, where $C(t)=\pi M^{2} \exp \left(\pi M^{2} t\right)$.

Proof. Applying (2.1) to $g(s)$ on the right-hand side of (2.1) and simplifying, we have

$$
\begin{aligned}
|g(t)| & \leq A(t)+M \int_{\delta}^{t} \frac{1}{\sqrt{t-s}}\left(A(s)+M \int_{\delta}^{s} \frac{1}{\sqrt{s-\tau}}|g(\tau)| d \tau\right) d s \\
& \leq a(t)+M^{2} \int_{\delta}^{t}\left(\int_{\delta}^{\tau} \frac{1}{\sqrt{t-s}} \frac{1}{\sqrt{s-\tau}} d s\right)|g(\tau)| d \tau
\end{aligned}
$$

by Tonelli's Theorem, where $a(t)=A(t)+M \int_{\delta}^{t}(\sqrt{t-s})^{-1} A(s) d s$. Therefore,

$$
|g(t)| \leq a(t)+\pi M^{2} \int_{\delta}^{t}|g(s)| d s \quad \text { a.e. on }[\delta, T]
$$

since

$$
\int_{s}^{t} \frac{1}{\sqrt{t-\tau}} \frac{1}{\sqrt{\tau-s}} d \tau=\pi \quad \text { for all } s \in[0, t) .
$$

Hence Gronwall's inequality gives

$$
\begin{aligned}
|g(t)| & \leq a(t)+\pi M^{2} \exp \left(\pi M^{2}(t-\delta)\right) \int_{\delta}^{t} a(s) d s \\
& =a(t)+\pi M^{2} \exp \left(\pi M^{2}(t-\delta)\right)\left(\int_{\delta}^{t} A(s) d s+2 M \int_{\delta}^{t} \sqrt{t-s} A(s) d s\right) \\
& \leq a(t)+\pi M^{2} \exp \left(\pi M^{2}(t-\delta)\right)(1+2 M \sqrt{t-\delta}) \int_{\delta}^{t} A(s) d s
\end{aligned}
$$

a.e. on $[\delta, T]$, which appears with $(2.2)$.

Now we prove (2.4). Let

$$
A(t)=B(t)+M \int_{0}^{\delta} \frac{1}{\sqrt{t-s}}|g(s)| d s .
$$


Inequality (2.3) becomes

$$
\begin{aligned}
|g(t)| \leq & A(t)+M \int_{\delta}^{t} \frac{1}{\sqrt{t-s}}|g(s)| d s \\
\leq & \left.A(t)+M \int_{\delta}^{t} \frac{1}{\sqrt{t-s}} A(s) d s+C(t, \delta) \int_{\delta}^{t} A(s) d s \quad \text { (by }(2.2)\right) \\
= & A(t)+M \int_{\delta}^{t} \frac{1}{\sqrt{t-s}} B(s) d s+M^{2} \int_{\delta}^{t} \int_{0}^{\delta} \frac{1}{\sqrt{t-s}} \frac{1}{\sqrt{s-\tau}}|g(\tau)| d \tau d s \\
& +C(t, \delta) \int_{\delta}^{t} B(s) d s+C(t, \delta) M \int_{\delta}^{t} \int_{0}^{\delta} \frac{1}{\sqrt{s-\tau}}|g(\tau)| d \tau d s \\
\leq & A(t)+M \int_{\delta}^{t} \frac{1}{\sqrt{t-s}} B(s) d s+\pi N^{2} \int_{0}^{\delta}|g(\tau)| d \tau+C(t, \delta) \int_{0}^{t} B(s) d s \\
& +2 M C(t, \delta)+M \int_{0}^{t}|g(\tau)| d \tau \quad(\text { by }(2.5)) \\
\rightarrow & B(t)+M \int_{0}^{t} \frac{1}{\sqrt{t-s}} B(s) d s+C(t) \int_{0}^{t} B(s) d s
\end{aligned}
$$

as $\delta \rightarrow 0$, since $g(\tau) / \sqrt{t-s}$ is integrable on $(0, t / 2]$ for a fixed $t$. Here $C(t)=\pi M^{2} \exp \left(\pi M^{2} t\right)$. We therefore obtain (2.4).

A straightforward calculation gives the following

Corollary 2.2. Suppose that the hypotheses of Lemma 2.1 hold with

$$
A(t)=M_{1}+\frac{M_{2}}{\sqrt{t}}+\frac{M_{3}}{t} \text { and } B(t)=M_{1}+\frac{M_{2}}{\sqrt{t}},
$$

where $M_{i} \geq 0, i=1,2,3$. Then we have

$$
|g(t)| \leq C_{1}(t, \delta) M_{1}+C_{2}(t, \delta) M_{2}+C_{3}(t, \delta) M_{3}
$$

a.e. on $[\delta, T]$. Here

$$
\begin{aligned}
& C_{1}(t, \delta)=1+2 \sqrt{t-\delta} M+(t-\delta) C(t, \delta), \\
& C_{2}(t, \delta)=\frac{1}{\sqrt{t}}+\pi M+2(\sqrt{t}-\sqrt{\delta}) C(t, \delta), \\
& C_{3}(t, \delta)=\frac{1}{t}+\sqrt{\frac{2}{t}} M(|\ln 2 t|+|\ln \delta|)+(|\ln t|+|\ln \delta|) C(t, \delta),
\end{aligned}
$$

and $C(t, \delta)=\pi M^{2} \exp \left(\pi M^{2}(t-\delta)\right)(1+2 M \sqrt{t-\delta})$.

Also, (2.4) gives

$$
|g(t)| \leq C_{4}(t) M_{1}+C_{5}(t) M_{2} \text { a.e. on }(0, T],
$$

where

$$
\begin{aligned}
& C_{4}(t)=1+2 \sqrt{t} M+t\left(\pi M^{2} \exp \left(\pi M^{2} t\right)\right), \\
& C_{5}(t)=\frac{1}{\sqrt{t}}+\pi M+2 \sqrt{t}\left(\pi M^{2} \exp \left(\pi M^{2} t\right)\right) .
\end{aligned}
$$

The main result of this section is the following theorem. 


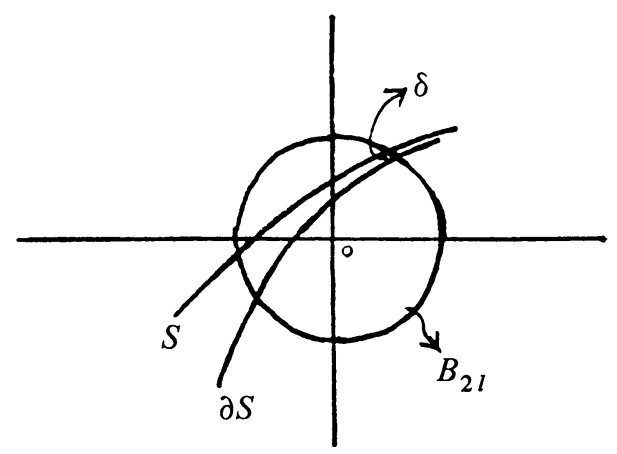

FIGURE 2.1

Theorem 2.3 (Existence and uniqueness of global solutions). Assume

(1) $f$ is a smooth Lipschitz vector function of class $C^{2}$ with Lipschitz constant $M$ in convex open set $S$. W.l.o.g., $0 \in S$ and $f(0)=0$.

(2) There is a convex open neighborhood $S^{\prime}$ of 0 such that $S^{\prime} \subset S$ and $\partial S \supset S$. Moreover, $S^{\prime}$ is invariant for the system (1.1)-(1.3).

(3) $u^{ \pm} \in S^{\prime}$ and $u_{0}(x) \in S^{\prime}$ almost all on $[-L, L]$ and $u_{0} \in L^{\infty}([-L, L])$. Then

(a) A unique $L^{\infty}([-L, L] \times[0, T])$ local solution $u$ of (1.8) exists. Moreover, $u(\cdot, t)$ is a $C^{2}$-function on $(-L, L)$ at each time level $t \in(0, T)$.

(b) $u(\cdot, t) \in C^{2}(-L, L)$ and satisfies (1.8) if and only if $u$ is a solution of the system (1.1)-(1.3) in the sense of $\S 1$.

(c) As long as $u$ satisfies (1.8) on $[0, T]$, then we have, for $t \leq T$,

$$
\mathrm{c}_{1}:\|u(t)\|_{\infty} \leq C(T)\left(\left\|u_{0}-h^{L}\right\|_{\infty}+\left\|h^{L}\right\|_{\infty}\right)
$$

for some constant $C(T)$ and

$$
\mathrm{c}_{2}:\|u(s)-u(t)\|_{\infty} \leq \frac{C}{t}(s-t)^{1 / 2} \text { for } 0<t<s \leq T
$$

for some constant $C=C\left(T, u_{0}, u^{ \pm} / L\right)$.

(d) A unique global solution of (1.8) exists and is a $C^{2}$-function on $(-L, L)$ at each time level $t>0$. Therefore by (b), the same is true. . $r$ the system (1.1)-(1.3) .

Proof. For notational simplicity, we let $q=\max \left(\left|u^{+}\right|,\left|u^{-}\right|\right)$and $l=$ $\left\|u_{0}-h^{L}\right\|_{\infty}+q$ and define $\delta=0$ if $B_{2 l} \subset S$, otherwise $\delta=\operatorname{dist}\left(\partial S^{\prime}, \partial S \cap B_{2 l}\right)$ $>0$, where $B_{2 l}$ is a ball with center at 0 and radius $2 l$ (see the figure). Let

$$
v(t)=G^{L}(t) *_{B}\left(u_{0}-h^{L}\right)+h^{L} .
$$

Then by means of the maximal principle, $v(x, t) \in S^{\prime}$, so that $v(x, t) \in B_{l} \cap S^{\prime}$. Now let

$$
\begin{aligned}
\mathscr{A}_{T}=\left\{u:[0, T] \rightarrow L^{\infty}([-L, L]) \mid \text { for all } t \in[0, T],\right. \\
\text { (i) } \left.\|u(t)\|_{\infty} \leq 2 l \text { and (ii) }\|u(t)-v(t)\|_{\infty} \leq \delta \text { if } \delta>0\right\},
\end{aligned}
$$


and define an operator $\mathscr{L}$ on $\mathscr{A}_{T}$ by

$$
\mathscr{L}(u)(t)=v(t)+\int_{0}^{t} G_{x}^{L}(t-s) *_{B} f(u(s)) d s .
$$

If $u \in \mathscr{A}_{T}$ then $u(x, t) \in B_{2 l}$ and $\|u-v\|_{\infty} \leq \delta$; hence from the fact that $v(x, t) \in B, \cap S^{\prime}$ and from the definition of $\delta$, we have $u(x, t) \in S$. By the bound (1.10) it is easy to show that, if $T$ is sufficiently small, then $\mathscr{L}$ maps $\mathscr{A}_{T}$ into itself and is contractive. Thus, there is a unique $u$ in $\mathscr{A}_{T}$ such that $u=\mathscr{L}(u)$.

To prove that $u(\cdot, t)$ is in $C^{2}(-L, L)$, we will require the following two facts, which we therefore prove first. Let $N=\left\|u_{0}-h^{L}\right\|_{\infty}+\left|u^{+}-u^{-}\right| / L$.

$\left(\mathbf{S}_{1}\right)$ If $w \in \mathscr{A}_{T}$ and $w(t) \in W^{2, \infty}$ with

$$
\left\|w_{x}(t)\right\|_{\infty} \leq \frac{2 C_{1}}{\sqrt{t}} N \quad \text { for all } t \in(0, T]
$$

and

$$
\left\|w_{x x}(t)\right\|_{\infty} \leq \frac{K_{0}}{\sqrt{t_{0}\left(t-t_{0}\right)}} N \text { for } 0<t_{0}<t \leq T
$$

where $C_{1}$ is a constant such that $\left\|G_{x}^{L}(t)\right\|_{1} \leq C_{1} / \sqrt{t}$ and $K_{0}>C_{1}\left(2 C_{1}+1\right)+2$, then $\mathscr{L}(W)$ satisfies (2.9) and (2.10) with some constants $C_{1}$ and $K_{0}$ if $T$ is sufficiently small.

$\left(\mathrm{S}_{2}\right)$ Given $\varepsilon_{0}>0$, let $\Omega_{T}\left(\varepsilon_{0}\right)=\left(-L+\varepsilon_{0}, L-\varepsilon_{0}\right) \times(0, T)$. If $w$ satisfies (2.9) and (2.10) then for $(x, t),(x, s) \in \Omega\left(\varepsilon_{0}\right)$,

$$
\left|\mathscr{L}(W)_{x}(x, s)-\mathscr{L}(w)_{x}(x, t)\right| \leq C \cdot \frac{N}{t}|s-t|^{1 / 2} .
$$

where $C=C\left(K_{0}, \varepsilon_{0}\right)$.

We prove $\left(S_{1}\right)$ in the following:

$$
\begin{aligned}
\left\|\mathscr{L}(w)_{x}(t)\right\|_{\infty} & \leq \frac{c_{1}}{\sqrt{t}}\left\|u_{0}-h^{L}\right\|_{\infty}+\frac{\left|u^{+}-u^{-}\right|}{2 L}+\int_{0}^{t} \frac{c_{1}}{\sqrt{t-s}} M \cdot 2 c_{1} N \frac{1}{\sqrt{s}} d s \\
& \leq \frac{2 C_{1}}{\sqrt{t}} N \text { for small } T .
\end{aligned}
$$

The semigroup property of $G^{L}$ implies that, for $t>t_{0}$,

$$
\begin{aligned}
\mathscr{L}(w)(t)= & G^{L}\left(t-t_{0}\right) *_{B}\left(w\left(t_{0}\right)-h^{L}\right)+h^{L} \\
& +\int_{t_{0}}^{t} G_{x}^{L}(t-s) *_{B} f(w(s)) d s .
\end{aligned}
$$


Thus by the fact that $G_{x x}^{L}=G_{y y}^{L}$ and integration by parts,

$$
\begin{aligned}
\left\|\mathscr{L}(w)_{x x}(t)\right\|_{\infty} \leq & \left\|G_{x}^{L}\left(t-t_{0}\right)\right\|_{1}\left\|w_{x}\left(t_{0}\right)-\frac{u^{+}-u^{-}}{2 L}\right\|_{\infty} \\
& \left.+\int_{t_{0}}^{t}\left\|G_{x}^{L}(t-s)\right\|_{1} \| f(w)(s)\right)_{x x} \|_{\infty} d s \\
& +\sup _{x} \int_{t_{0}}^{t}\left|G_{x}^{L}(\cdot, \pm L, t-s)\right|\left\|f(w(s))_{x}\right\|_{\infty} d s .
\end{aligned}
$$

However, for $s \geq t_{0}$,

$$
\begin{aligned}
\left\|f(w(s))_{x x}\right\|_{\infty} & \leq\left\|D^{2} f\right\|_{\infty, T}\left\|w_{x}(s)\right\|_{\infty}^{2}+M\left\|w_{x x}(s)\right\|_{\infty} \\
& \leq\left\|D^{2} f\right\|_{\infty, T}\left(\frac{2 c_{1}}{\sqrt{s}} N\right)^{2}+M \cdot \frac{K_{0}}{\sqrt{t_{0}\left(s-t_{0}\right)}} N,
\end{aligned}
$$

and we have

$$
\begin{aligned}
& \int_{t_{0}}^{t}\left\|G_{x}^{L}(t-s)\right\|_{1}\left\|f(w(s))_{x x}\right\| d s \\
& \quad \leq 4 c_{1}^{2} N^{2}\left\|D^{2} f\right\|_{\infty, T} \int_{t_{0}}^{t} \frac{c_{1}}{\sqrt{t-s}} \frac{1}{s} d s+M K_{0} N \pi \frac{1}{\sqrt{t_{0}}} \\
& \quad \leq k_{0} N \sqrt{\frac{2}{t}}\left(|\ln t|+\left|\ln t_{0}\right|\right)+K_{0} M N \pi \frac{1}{\sqrt{t_{0}}}
\end{aligned}
$$

by (2.5) and the bound

$$
\int_{t_{0}}^{t} \frac{1}{\sqrt{t-s} s} d s \leq \sqrt{\frac{2}{t}}\left(|\ln t|+\left|\ln t_{0}\right|\right)
$$

where $k_{0}=4 c_{1}^{2} N\left\|D^{2} f\right\|_{\infty, T}$. The R.H.S. above is then bounded by

$$
4 k_{0} N \frac{1}{\sqrt{t-t_{0}}}\left|\ln t_{0}\right|+\frac{1}{2} \frac{1}{\sqrt{t_{0}\left(t-t_{0}\right)}} N \leq \frac{N}{\sqrt{t_{0}\left(t-t_{0}\right)}}
$$

for small $T$ since $\sqrt{t}_{0}\left|\ln t_{0}\right| \rightarrow 0$ as $t_{0} \rightarrow 0$. Moreover, using the bound (see Ladyzhenskaya [10])

$$
\left|G_{x}(x, \pm L, t-s)\right| \leq \frac{c^{\prime}}{(t-s)^{3 / 2}}|x \pm L| \exp \left(-\frac{(x \pm L)^{2}}{4 \mu(t-s)}\right)
$$

we have

$$
\begin{aligned}
& \int_{t_{0}}^{t}\left|G_{x}^{L}(\cdot, \pm L, t-s)\right|\left\|f(w(s))_{x}\right\|_{\infty} d s \\
& \quad \leq 2 c_{1} M N c^{\prime} \int_{t_{0}}^{t} \frac{1}{(t-s)^{3 / 2} \sqrt{s}}|x \pm L| \exp \left(-\frac{(x \pm L)^{2}}{4 \mu(t-s)}\right) d s \\
& \quad \leq K_{1} \mu^{1 / 2} \frac{N}{\sqrt{t_{0}}} \int_{0}^{\infty} \exp \left(-\tau^{2}\right) d \tau,
\end{aligned}
$$


where $K_{1}=2 c_{1} M c^{\prime}$ and $\tau=(x \pm L) / 2 \sqrt{\mu(t-s)}$. The right-hand side above is therefore bounded by

$$
K_{1} \mu^{1 / 2} N \frac{1}{\sqrt{t_{0}}} \leq \frac{1}{\sqrt{t_{0}\left(t-t_{0}\right)}} N \text { for small } T .
$$

Therefore, from (2.11) it follows that

$$
\begin{aligned}
\left\|\mathscr{L}(w)_{x x}(t)\right\|_{\infty} & \leq \frac{c_{1}}{\sqrt{t-t_{0}}}\left(\frac{2 c_{1}}{\sqrt{t_{0}}} N+N\right)+\frac{2 N}{\sqrt{t_{0}\left(t-t_{0}\right)}} \\
& \leq \frac{K_{0}}{\sqrt{t_{0}\left(t-t_{0}\right)}} N .
\end{aligned}
$$

Next, we prove $\left(\mathrm{S}_{2}\right)$. By Duhamel's principle, $\mathscr{L}(w)$ is the solution of

$$
\left\{\begin{array}{l}
U_{t}=D U_{x x}-f(w)_{x} \text { on } \Omega_{T}, \\
U( \pm L, t)=u^{ \pm} \\
U(x, 0)=u_{0}(x)
\end{array} \quad t \in(0, T)\right.
$$

Let $V \equiv \mathscr{L}(w)$ on $\bar{\Omega}(0)$ and $\equiv 0$ otherwise. Then $V_{t}=D V_{x x}-f(w)_{x}$ on $\Omega_{T}(0)$. Given $\varepsilon>0$, let $j_{\varepsilon}$ be the standard mollifier with support in $[-\varepsilon, \varepsilon]$. We adopt the notation

$$
V^{\ell}=j_{\varepsilon} * V \text { and } F^{\varepsilon}=j_{\varepsilon} * f(w)
$$

Then

$$
\left(V^{\varepsilon}\right)_{t}=\mu\left(V^{\varepsilon}\right)_{x x}-\left(F^{\varepsilon}\right)_{x} \text { on } \Omega(\varepsilon)
$$

so that

$$
\left(V_{x}^{\varepsilon}\right)_{t}=\mu\left(V_{x}^{\varepsilon}\right)_{x x}-\left(F_{x}^{\varepsilon}\right)_{x} \text { on } \Omega(\varepsilon) .
$$

From the inequality (2.10) with $t_{0}=t / 2$ for $\mathscr{L}(w)$, we have $\left\|V_{x x}(t)\right\|_{\infty} \leq$ $2 K_{0} N / t$. Therefore, if $(x, t),(x, s) \in \Omega(\varepsilon)$ and $t<s$, then

$$
\begin{aligned}
\left|V_{x}^{\varepsilon}(x, s)-V_{x}^{\varepsilon}(x, t)\right| & \left.=\left|V_{x t}^{\varepsilon}\left(x, t^{\prime}\right)\right|(s-t) \quad \text { where } t^{\prime}=t^{\prime}(x) \in(t, s)\right) \\
& =\left|V_{x x x}^{\varepsilon}\left(x, t^{\prime}\right)-F_{x x}^{\varepsilon}\left(x, t^{\prime}\right)\right|(s-t) \\
& =\left|j_{\varepsilon}^{\prime} * V_{x x}\left(x, t^{\prime}\right)-j_{\varepsilon}^{\prime} * f(w)_{x}\left(x, t^{\prime}\right)\right|(s-t) \\
& \leq\left\|j_{\varepsilon}^{\prime}\right\|_{1}\left(\left\|V_{x x}\left(t^{\prime}\right)\right\|_{\infty}+\left\|f(w)_{x}\left(t^{\prime}\right)\right\|_{\infty}\right)(s-t) \\
& \leq \frac{c}{\varepsilon}\left(\frac{2 K_{0}}{t^{\prime}} N+M \frac{2 c_{1}}{\sqrt{t}} N\right)(s-t) \quad(\text { by }(2.9)) \\
& \leq \frac{c\left(K_{0}\right)}{t} \cdot \frac{s-t}{\varepsilon} N .
\end{aligned}
$$


Moreover, if $(x, t),(x, s) \in \Omega_{T}\left(\varepsilon_{0}\right), t<s$, and $\varepsilon \leq \varepsilon_{0}$ (so that $\Omega_{T}\left(\varepsilon_{0}\right) \subset$ $\left.\Omega_{T}(\varepsilon)\right)$, then

$$
\begin{aligned}
\mid V_{x}(x, s) & -V_{x}(s, t)|\leq| \int_{|z| \leq 1} j(z)\left(V_{x}(x, s)-V_{x}(x-\varepsilon z, s)\right) d z \mid \\
& \quad+\left|V_{x}^{\varepsilon}(x, s)-V_{x}^{\varepsilon}(x, t)\right|+\left|\int_{|z| \leq 1} j(z)\left(V_{x}(x-\varepsilon z, t)-V_{x}(x, t)\right) d z\right| \\
\leq & \left\|V_{x x}(s)\right\|_{\infty} \varepsilon+\frac{c(K)}{t} \frac{s-t}{\varepsilon} N+\left\|V_{x x}(t)\right\| \varepsilon \\
\leq & \frac{c\left(K_{0}\right)}{t} N\left(\varepsilon+\frac{s-t}{\varepsilon}\right) \quad\left(\text { for all } \varepsilon \leq \varepsilon_{0}\right) \\
= & \left.\frac{c\left(K_{0}\right)}{t} N \cdot 2(s-t)^{1 / 2} \quad \text { (if we take } \varepsilon=(s-t)^{1 / 2} \leq \varepsilon_{0}\right) .
\end{aligned}
$$

Therefore, there is a constant $C\left(K_{0}, \varepsilon_{0}\right)$ such that $\left(\mathrm{S}_{2}\right)$ holds.

Now, to prove that $u(t) \in C^{2}(-L, L)$, we let $w^{0}=0$ and $w^{n}=\mathscr{L}\left(w^{n-1}\right)$. Then for all $n \geq 0$, by (2.9) and (2.10), we have, for $0<t<T$,

$$
\left\|w_{x}^{n}(t)\right\|_{\infty}=\left\|\mathscr{L}\left(w^{n-1}\right)_{x}(t)\right\|_{\infty} \leq \frac{2 c_{1}}{\sqrt{t}} N
$$

and

$$
\left\|w_{x x}^{n}(t)\right\|_{\infty}=\left\|\mathscr{L}\left(w^{n-1}\right)_{x x}(t)\right\|_{\infty} \leq \frac{2 K}{t} N
$$

(by taking $t_{0}=t / 2$ ), where $c_{1}$ and $K$ are independent of $n$ and $t$.

Thus $w^{n} \rightarrow u$ in $L^{\infty}$ since $\mathscr{L}$ is contractive and $u=\mathscr{L}(u)$ with $u$ uniquely determined. By $\left(\mathbf{S}_{2}\right)$ and (2.14), $\left\{w_{x}^{n}\right\}$ are uniformly Hölder continuous with exponent $\frac{1}{2}$ on any $\Omega \subset \subset \Omega_{T}$; and for each $n \geq 1, w^{n}$ is the solution of $U_{t}=\mu U_{x x}-f(w)_{x}$ on $\Omega$. Hence the standard Schauder theory for a parabolic equation implies that $\left\{w_{x x}^{n}\right\}$ are uniformly Hölder continuous on $\Omega$. Therefore, for each $t>0,\left\{w_{x x}^{n}(t)\right\}$ is an equicontinuous family of functions from $(-L, L)$ to $\mathbf{R}^{m}$ and $\left\{w_{x x}^{n}(x, t): n \geq 0\right\}$ is uniformly bounded independently of $n$ and $x$ by (2.14) and the continuity of $w_{x x}^{n}(t)$. Therefore by Ascoli's theorem, there is a subsequence $\left\{w_{x x}^{n_{j}}(t)\right\}$ which converge pointwise (uniformly converges on every compact subset of $(-L, L))$. Moreover, for each $t, w^{n}(t) \rightarrow u(t)$ in $L^{\infty}(-L, L)$. Hence $w^{n}(t) \rightarrow u(t)$ in $\mathscr{D}(-L, L)$ so that $w_{x x}^{n}(t) \rightarrow u_{x x}(t)$ in $\mathscr{D}(-L, L)$, too. Therefore $u_{x x}(t)=w(t)$ is continuous. Thus, we have shown that there is a unique solution $u=\mathscr{L}(u)$ and for each $t, u(t) \in C^{2}(-L, L)$.

(b) Both implications can be shown to hold by Duhamel's principle.

$\left(c_{1}\right)$ Assume $u$ satisfies (1.8). Then

$$
\|u(t)\|_{\infty} \leq\left\|u_{0}-h^{L}\right\|_{\infty}+\left\|h^{L}\right\|_{\infty}+\int_{0}^{t} \frac{c_{1}}{\sqrt{t-s}} M\|u(s)\|_{\infty} d s
$$


If $\|u(t)\|_{\infty}$ is integrable then (2.8) with $M_{2}=0$ gives $\left(\mathrm{c}_{1}\right)$. To prove that $\|u(t)\|_{\infty}$ is integrable on $[0, T]$, we integrate both sides of $(2.15)$ on $[0, t]$ and let $N=\left\|u_{0}-h^{L}\right\|_{\infty}+\left\|h^{L}\right\|_{\infty}$ to obtain

$$
\begin{aligned}
\int_{0}^{t}\|u(s)\|_{\infty} d s & \leq t N+C_{1} M \int_{0}^{t} \int_{0}^{s} \frac{1}{\sqrt{s-\tau}}\|u(\tau)\| d \tau d s \\
& =t N+C M \int_{0}^{t} \sqrt{t-\tau}\|u(\tau)\|_{\infty} d \tau \quad \text { (by Tonelli's theorem) } \\
& \leq t N+\sqrt{t} C_{1} M \int_{0}^{t}\|u(\tau)\|_{\infty} d \tau .
\end{aligned}
$$

Hence if $\sqrt{T_{0}} C_{1} M \leq \frac{1}{2}$ and $0 \leq t \leq T_{0}$ then we have

$$
\int_{0}^{t}\|u(s)\|_{\infty} d s \leq 2 t N
$$

In addition, given $t \in\left[k T_{0},(k+1) T_{0}\right]$ for some integer $k, u(t)$ is the solution of (1.8) with initial data $u\left(k T_{0}\right)$ at time $t-k T_{0}$; this implies $\int_{k T_{0}}^{(k+1) T_{0}}\|u(s)\|_{\infty} d s$ $<\infty$. Therefore, $\|u(t)\|_{\infty}$ is integrable on $[0, T]$ (one can use the same technique to prove that $\left\|u_{x}(t)\right\|_{\infty}$ and $\left\|u_{x x}(t)\right\|_{\infty}$ are integrable on $\left.[0, T]\right)$.

$\left(c_{2}\right)$ From (1.8), we have

$$
\left\|u_{x}(t)\right\|_{\infty} \leq \frac{c_{1}}{\sqrt{t}}\left\|u_{0}-h^{L}\right\|_{\infty}+\frac{\left|u^{+}-u^{-}\right|}{2 L}+c_{1} M \int_{0}^{t} \frac{1}{\sqrt{t-s}}\left\|u_{x}(s)\right\|_{\infty} d s .
$$

Thus, by Corollary 2.2 ,

$$
\left\|u_{x}(t)\right\|_{\infty} \leq \frac{C(T)}{\sqrt{t}}\left(\left\|u_{0}-h^{L}\right\|_{\infty}+\frac{\left|u^{+}-u^{-}\right|}{2 L}\right) \equiv g(t) .
$$

Hence for all $h>0$,

$$
\begin{aligned}
\mid u(x, s) & -u(x, t) \mid \\
\leq & \frac{1}{h}\left|\int_{x}^{x+h} u(y, s)-u(y, t) d y\right|+h\left(\left\|u_{x}(t)\right\|_{\infty}+\left\|u_{x}(s)\right\|_{\infty}\right) \\
\leq & \frac{1}{h}\left|\int_{x}^{x+h} \int_{t}^{s} u_{t}(y, \tau) d \tau d y\right|+2 g(t) h \\
= & \frac{1}{h}\left|\int_{t}^{s} \int^{x+h}-u_{y y}(y, \tau)-f(u(y, \tau))_{y} d y d \tau\right|+2 g(t) h \\
\leq & \frac{2}{h} \int_{t}^{s}\left(\left\|u_{x}(\tau)\right\|_{\infty}+M\|u(\tau)\|_{\infty}\right) d \tau+2 g(t) h \\
\leq & \frac{2}{h} \int_{t}^{s} g(\tau) d \tau+\frac{2}{h} M C(T)\left(\left\|u_{0}-h^{L}\right\|_{\infty}+\left\|h^{L}\right\|_{\infty}\right)(s-t)+2 g(t) h \\
\leq & \frac{1}{t} C\left(T, u_{0}, u^{ \pm}\right)\left(\frac{s-t}{h}+h\right) .
\end{aligned}
$$


$\left(c_{2}\right)$ is then proved by taking $h=(s-t)^{1 / 2}$.

(d) To prove the existence of the unique global solution, we let $A=\{t>0$ : there is a unique solution of $(1.1)-(1.3)$ on $[0, t)\}$. Clearly, $A$ is closed and nonempty by (a). To prove that $A$ is open, we take $t_{0} \in A$. Then for $t \in\left(0, t_{0}\right)$

$$
\begin{aligned}
u(t)= & G^{L}(t) *_{B}\left(u_{0}-h^{L}\right)+h^{L} \\
& -\int_{0}^{t} G^{L}(t-s){ }_{B} f(u(s))_{x} d s .
\end{aligned}
$$

Take a sequence $\left\{t_{n}\right\}$ such that $t_{n} \uparrow t_{0}$; then $\left\{u\left(t_{n}\right)\right\}$ is a Cauchy sequence in $C([-L, L])$ by $\left(\mathrm{c}_{2}\right)$. Define $u$ at $t_{0}$ by the limit of $u\left(t_{n}\right)$. Then, replacing $t$ by $t_{n}$ in (2.16) and taking the limit in $C([-L, L])$, we have (2.16) for $t=t_{0}$ and $u\left(t_{0}\right) \in S$ since $u\left(t_{n}\right) \in S^{\prime} \subset S \quad \forall n \geq 1$. Applying the local existence theorem (a) with initial data $u\left(t_{0}\right)$, we can find $\delta>0$ such that, for $t \in\left(t_{0}, t_{0}+\delta\right)$,

$$
\begin{aligned}
u(t)= & G^{L}\left(t-t_{0}\right) *_{B}\left(u\left(t_{0}\right)-h^{L}\right)+h^{L} \\
& -\int_{t_{0}}^{t} G^{L}(t-s){ }_{B} f(u(s))_{x} d s
\end{aligned}
$$

and $u(t)$ is $C^{2}(-L, L)$. Substitute (2.16) for $u\left(t_{0}\right)$ in (2.17). The semigroup property of $G^{L}(t)$ then implies that the expression (1.8) holds for all $t \in\left(0, t_{0}+\right.$ $\delta$ ) and that $u(t)$ is a $C^{2}(-L, L)$ function for all $t \in\left(0, t_{0}+\delta\right) \backslash\left\{t_{0}\right\}$. It remains to show that $u\left(t_{0}\right)$ is a $C^{2}(-L, L)$ function. For each $n, u\left(t_{n}\right)$ satisfies

$$
\begin{aligned}
u\left(t_{n}\right)= & G^{L}\left(t_{n}-t_{1}\right) *_{B}\left(u\left(t_{1}\right)-h^{L}\right)+h^{L} \\
& -\int_{t_{1}}^{t} G^{L}(t-s) *_{B} f(u(s))_{x} d s,
\end{aligned}
$$

where $u\left(t_{1}\right)$ is $C^{2}(-L, L)$. By the same argument as that occurring in the proof of $\left(c_{2}\right)$, we can show

$$
\left\|u_{x}\left(t_{n}\right)\right\|_{\infty} \leq C\left(t_{1}\right) \quad \forall n \geq 1
$$

and

$$
\left\|u_{x x}\left(t_{n}\right)\right\|_{\infty} \leq C^{\prime}\left(t_{1}\right) \quad \forall n \geq 1
$$

In addition, the proof of $\left(\mathrm{S}_{2}\right)$ in part $(\mathrm{a})$ with the initial time 0 replaced by $t_{0}$ gives

$$
\left\|u_{x}\left(t_{l}\right)-u_{x}\left(t_{k}\right)\right\|_{\infty} \leq \frac{\text { const. }}{t_{1}}\left|t_{l}-t_{k}\right|^{1 / 2}
$$

The fact that $u\left(t_{0}\right) \in C^{2}(-L, L)$ then follows as in the last part of the proof of $(\mathbf{a})$.

Therefore, by (b), there is a solution of $(1.1)-(1.3)$ on $\left(0, t_{0}+\delta\right)$. Thus $A$ is open.

The next theorem is about estimating the bounds for the derivatives of the exact solution $v(t)$ of $(1.1)-(1.3)$. 
Theorem 2.4. Suppose $v(t)$ is the exact solution of (1.1)-(1.3), and let $l_{\infty}=$ $\left\|u_{0}-h^{L}\right\|_{\infty}, l_{ \pm}=\left|u^{+}-u^{1}\right| / L$. If $u_{0}$ is in $L^{\infty}(-L, L)$, then for all $t \in(0, T]$,

(a) $\left\|v_{x}(t)\right\|_{\infty} \leq C(T)\left(l_{ \pm}+l_{\infty} / \sqrt{t}\right)$;

(b) $\left\|v_{x x}(t)\right\|_{\infty} \leq C(T)\left[l_{ \pm}^{2}+l_{ \pm}+\left(l_{\infty}^{2}+l_{\infty}+l_{ \pm}\right) / \sqrt{t}+l_{\infty} / t\right]$.

Note that all the constants $C(T)$ depend on $T$ and $\mu_{i}, i=1, \ldots, m$, and the Lipschitz constant $M$ of $f$.

Proof. (a) From the representation (1.8)

$$
\begin{aligned}
v_{x}(x, t)= & \int_{-L}^{L} G_{x}^{L}(x, y, t)\left(u_{0}-h^{L}\right)(y) d y+\frac{1}{2} \frac{u^{+}-u^{-}}{L} \\
& -\int_{0}^{t} \int_{-L}^{L} G_{x}^{L}(x, y, t-s) f(v(y, s))_{y} d y d s
\end{aligned}
$$

so that

$$
\left\|V_{x}(t)\right\|_{\infty} \leq \frac{c_{1}}{\sqrt{t}} l_{\infty}+\frac{1}{2} l_{ \pm}+\int_{0}^{t} c_{1} M \frac{1}{\sqrt{t-s}}\left\|v_{s}(s)\right\|_{\infty} d s .
$$

This is a Gronwall-type inequality, so that by Corollary 2.2 we have

$$
\begin{aligned}
\left\|v_{x}(t)\right\|_{\infty} & \leq C(T)\left(l_{ \pm}+l_{\infty}\right)+C(T) \frac{1}{\sqrt{t}} \\
& =C(T)\left(l_{ \pm}+\frac{1}{\sqrt{t}} l_{\infty}\right) .
\end{aligned}
$$

(b) The semigroup property of $G^{L}$ implies that given $s_{0}$ and $t \in(0, T]$ we have

$$
\begin{aligned}
v\left(s_{0}+t\right)= & G^{L}(t) *_{B}\left(v\left(s_{0}\right)-h^{L}\right)+h^{L} \\
& -\int_{0}^{t} G^{L}(t-s) *_{B} f\left(v\left(s_{0}+s\right)\right)_{x} d s
\end{aligned}
$$

Hence by the fact that $G_{x x}^{L}=G_{y y}^{L}$ and $G^{L}(x, \pm L, t)=0$, which follows by integration by parts, we have

$$
\begin{aligned}
v_{x x}\left(s_{0}+t\right)= & \int_{-L}^{L} G_{x}^{L}(x, y, t)\left(v_{x}\left(s_{0}\right)-\frac{u^{+}-u^{-}}{2 L}\right) d y \\
& -\int_{0}^{t} \int_{-L}^{L} G_{y}^{L}(x, y, t-s) f\left(v\left(y, s_{0}+s\right)\right)_{y y} d y d s \\
& +\left.\int_{0}^{t} G_{y}^{L}(x, \cdot, t-s) f\left(v\left(\cdot, s_{0}+s\right)\right)_{y}\right|_{-L} ^{L} d s .
\end{aligned}
$$


It follows from (2.12) and the result of (a) that

$$
\begin{aligned}
&\left\|v_{x x}\left(x_{0}+t\right)\right\|_{\infty} \\
& \leq \frac{c_{1}}{\sqrt{t}}\left(\left\|v_{x}\left(s_{0}\right)\right\|_{\infty}+\frac{\left|u^{+}-u^{-}\right|}{2 L}\right) \\
&+\int_{0}^{t} \frac{c_{1}}{\sqrt{t-s}}\left(\left\|D^{2} f\right\|_{\infty, T}\left\|v_{x}\left(s_{0}+s\right)\right\|_{\infty}^{2}+M\left\|v_{x x}\left(s_{0}+s\right)\right\|_{\infty}\right) d s \\
&+C(T) \frac{c_{1}}{\sqrt{t}} \frac{1}{\sqrt{s_{0}}}\left(\left\|u_{0}-h^{L}\right\|_{\infty}+\frac{\left|u^{+}-u^{-}\right|}{2 L}\right) \\
& \leq \frac{C(T)}{\sqrt{t}}\left(l_{ \pm}+\frac{1}{\sqrt{s_{0}}} l_{\infty}\right)+C(T) \int_{0}^{t} \frac{1}{\sqrt{t-s}}\left(l_{ \pm}+\frac{1}{\sqrt{s_{0}+s}} l_{\infty}\right)^{2} d s \\
&+C_{1} M \int_{0}^{t} \frac{1}{\sqrt{t-s}}\left\|v_{x x}\left(s_{0}+s\right)\right\|_{\infty} d s .
\end{aligned}
$$

The second term is bounded by

$$
\begin{aligned}
C(T) & \int_{0}^{t} \frac{1}{\sqrt{(t-s)}}\left(l_{ \pm}^{2}+\frac{1}{s_{0}+s} l_{\infty}^{2}\right) d s \\
& \leq C(T) \sqrt{t} l_{ \pm}^{2}+C(T) l_{\infty}^{2} \frac{1}{\sqrt{s_{0}}} \int_{0}^{t} \frac{1}{\sqrt{t-s}} \frac{1}{\sqrt{s}} d s \\
& =C(T) l_{ \pm}^{2}+C(T) l_{\infty}^{2} \frac{1}{\sqrt{s_{0}}}
\end{aligned}
$$

by $1 /\left(s_{0}+s\right) \leq 1 / \sqrt{s_{0}} \sqrt{s}$ and (2.12). The above inequality becomes

$$
\begin{aligned}
\left\|v_{x x}\left(s_{0}+t\right)\right\|_{\infty} \leq & C(T)\left(l_{ \pm}^{2}+\frac{1}{\sqrt{s_{0}}} l_{\infty}^{2}\right)+C(T) \frac{1}{\sqrt{t}}\left(l_{ \pm}+\frac{1}{\sqrt{s_{0}}} l_{\infty}\right) \\
& +C_{1} M \int_{0}^{t} \frac{1}{\sqrt{t-s}}\left\|v_{x x}\left(s_{0}+s\right)\right\|_{\infty} d s .
\end{aligned}
$$

This is a Gronwall-type inequality. Hence by Corollary 2.2 we have

$$
\left\|v_{x x}\left(s_{0}+t\right)\right\|_{\infty} \leq C(T)\left[l_{ \pm}^{2}+\frac{1}{\sqrt{s_{0}}} l_{\infty}^{2}+\left(l_{ \pm}+\frac{1}{\sqrt{s_{0}}} l_{\infty}\right)+\frac{1}{\sqrt{t}}\left(l_{ \pm}+\frac{1}{\sqrt{s_{0}}} l_{\infty}\right)\right] .
$$

Thus, given any $t_{0} \in(0, T],(\mathrm{b})$ is obtained by taking $t=s_{0}=t_{0} / 2$ in the above inequality.

We remark that one can prove the existence of classical solutions of the Cauchy problem (1.1)-(1.2) by exactly the same proof as that of Theorem 2.3, but using the representation (1.7). The estimates of Theorem 2.4 hold for the solution of $(1.1)-(1.2)$ with $\left|u^{+}-u^{-}\right|=0$ and $h^{L}=0$.

\section{Convergence Rates}

We assume throughout this section that $u$ and $u^{L}$ are the exact solutions of (1.1)-(1.2) and (1.1)-(1.3) respectively. Also we adopt the notations 
$\mu^{\prime}=\min \left(\mu_{1}, \ldots, \mu_{m}\right), \mu=\|D\|_{\infty}$, and

$$
\left\|u(t)-u^{L}(t)\right\|_{\infty, L}=\sup _{x \in[-L, L]}\left|u(x, t)-u^{L}(x, t)\right| .
$$

Our main result in this section concerns a bound for $\left\|u(t)-u^{L}(t)\right\|_{\infty, L}$ in terms of the initial data and $1 / L^{j}$.

Lemma 3.1. For $|x| \leq L$, we have

$$
\int_{|y|>L} K(x-y, t) d y \leq \frac{1}{2}\left(\exp \left(-\frac{L^{2}}{4 \mu t}\right)+\exp \left(-\frac{L-|x|^{2}}{4 \mu t}\right)\right)
$$

and

$$
\int_{|y|>L}\left|K_{y}(x-y, t)\right| d y \leq \frac{2}{\sqrt{\pi \mu^{\prime} t}} \exp \left(-\frac{L-|x|^{2}}{4 \mu t}\right) .
$$

Proof. By the bound

$$
\int_{|x|>L} \exp \left(-(x-l)^{2}\right) d x \leq \frac{\sqrt{\pi}}{2}\left[\exp \left(-L^{2}\right)+\exp (-L-|l|)^{2}\right]
$$

and simple computation, one can prove (3.1) and (3.2).

Lemma 3.2. Given any real numbers $u^{+}$and $u^{-}$, a bounded function $g$ on $\mathbf{R}$, and a number $L>0$, we have

(i) $\left\|K(t) * g-u^{ \pm}\right\|_{\infty,[ \pm 2 L, \pm \infty)} \leq W_{ \pm}^{L}\left(g, u^{+}, t\right)$,

(ii) $\left\|K_{x}(t) * g\right\|_{\infty,[ \pm 2 L, \pm \infty)} \leq Z_{ \pm}^{L}\left(g, u^{ \pm}, t\right)$,

where $g^{ \pm}=\frac{1}{2}\left\|g-u^{ \pm}\right\|_{\infty}, l_{e}=\exp \left(-L^{2} / 4 \mu t\right)$,

$$
\begin{aligned}
& W_{ \pm}^{l}\left(g, u^{ \pm}, t\right)=\left\|g(\cdot)-u^{ \pm}\right\|_{\infty,[ \pm L, \pm \infty)}+g^{ \pm} l_{e}, \\
& Z_{ \pm}^{L}\left(g, u^{ \pm}, t\right)=\frac{c_{1}}{\sqrt{t}}\left\|g(\cdot)-u^{ \pm}\right\|_{\infty,[ \pm L, \pm \infty)}+\frac{1}{\sqrt{\pi \mu^{\prime} t}} g^{ \pm} l_{e},
\end{aligned}
$$

and $c_{1}$ is the constant such that $\left\|K_{x}(t)\right\|_{1} \leq c_{1} / \sqrt{t}$.

Proof. (i) If $x \geq 2 L$, then by Lemma 3.1,

$$
\begin{aligned}
\mid K(\cdot, t) & * g-u^{+}|=| K(\cdot, t) *\left(g-u^{+}\right) \mid \\
& \leq \int_{-\infty}^{L} K(y, t)\left|g(x-y)-u^{+}\right| d y+\int_{L}^{\infty} K(y, t)\left|g(x-y)-u^{+}\right| d y \\
& \leq\left\|g(\cdot)-u^{+}\right\|_{\infty,[L, \infty)}+\left\|g-u^{+}\right\|_{\infty} \cdot \frac{1}{2} \exp \left(-\frac{L^{2}}{4 \mu t}\right) .
\end{aligned}
$$

For $x \leq-2 L$, the result follows from the same proof as above.

(ii) If $x \geq 2 L$, then by Lemma 3.1 . 


$$
\begin{aligned}
& \left|K_{x}(t) * g\right|=\left|K_{x}(t) * g-K_{x}(T) * u^{+}\right|=\left|K_{x}(t) *\left(g-u^{+}\right)\right| \\
& \quad \leq \int_{-\infty}^{L}\left|K_{y}(x-y, t)\left(g(y)-u^{+}\right)\right| d y+\int_{L}^{\infty}\left|K_{y}(x-y, t)\left(g(y)-u^{+}\right)\right| d y \\
& \quad \leq\left\|g-u^{+}\right\| \frac{1}{\sqrt{\pi \mu^{\prime} t}} \exp \left(-\frac{L^{2}}{4 \mu t}\right)+\frac{c_{1}}{\sqrt{t}}\left\|g-u^{+}\right\|_{\infty,[L, \infty)} .
\end{aligned}
$$

For $x \leq-2 L$, the result follows from the same proof as above.

Lemma 3.3. Let

$$
z(x, t)=K(t) * u_{0}-\left(G^{2 L}(t) *_{B}\left(u_{0}-h^{2 L}\right)+h^{2 L}\right)
$$

Then

$$
\|z\|_{\infty, \Omega_{2 L}(t)} \leq \max \left(W_{+}^{L}\left(u_{0}, u^{+}, t\right), W_{-}^{L}\left(u_{0}, u^{-}, t\right)\right),
$$

where $\Omega_{2 L}(t)=[-2 L, 2 L] \times[0, t)$ and $h^{2 L}$ is defined in (1.8).

Proof. $z(x, t)$ satisfies

$$
\begin{cases}z_{t}=D z_{x x} & \text { on }(-2 L, 2 L) \times(0, \infty), \\ z(x, 0)=0 & \text { on }[-2 L, 2 L], \\ z( \pm 2 L, t)= & K(t) * u_{0}( \pm 2 L)-u^{ \pm}\end{cases}
$$

Hence by the maximum principle and Lemma 3.2, we have

$$
\|z\|_{\infty, \Omega_{2 L}(t)} \leq \sup _{\partial^{\prime} \Omega}|z(x, s)| \leq \max _{0 \leq s \leq t}\left(W_{+}^{L}\left(u_{0}, u^{+}, s\right), W_{-}^{L}\left(u_{0}, u^{-}, s\right)\right),
$$

where $\partial^{\prime} \Omega=\{( \pm L, s)$ or $(x, 0)|0 \leq s<t| x \mid, \leq 2 L\}$. Thus the continuity of $z(x, t)$ implies that

$$
|z|_{\infty, \Omega_{2 L}(t)} \leq \max \left(W_{+}^{L}\left(u_{0}, u^{+}, t\right), W_{-}^{L}\left(u_{0}, u^{-}, t\right)\right)
$$

The following lemma is needed for the proof of the next theorem, which is the main result in this section.

\section{Lemma 3.4.}

$$
\begin{gathered}
\left\|\int_{0}^{t} K(t-s) * f(u(s))_{x} d s-\int_{0}^{t} G^{2 L}(t-s){ }_{B} f(u(s))_{x} d s\right\|_{\infty,[-2 L, 2 L]} \\
\leq M \int_{0}^{t}\left\|u_{x}(s)\right\|_{\infty,|x| \geq L} d s+C(T)\left\|u_{0}\right\|_{\infty} \exp \left(-\frac{L^{2}}{4 \mu t}\right),
\end{gathered}
$$

where $C(T)$ does not depend on $L$.

Proof. Fix $\tau$ and $t$ so that $t-\tau>0$. Let

$$
z(x, s)=K(s) * f(u(\tau))_{x}-G^{2 L}(s){ }_{B} f(u(\tau))
$$


for $s \in(0, t-\tau)$ and $|x| \leq 2 L$. Then $z(x, s)$ satisfies

$$
\left\{\begin{array}{l}
z_{t}=D z_{x x} \text { on }(-2 L, 2 L) \times(0, t-\tau), \\
z( \pm 2 L, s)=K(s) * f(u(\tau))_{x}( \pm 2 L) \text { for } s \in(0, t-\tau), \\
z(x, 0)=0 \text { for }|x| \leq 2 L .
\end{array}\right.
$$

Therefore, by the maximal principle,

$$
|z(x, s)| \leq \sup _{0 \leq s^{\prime}<t-\tau}\left|K\left(s^{\prime}\right) * f(u(\tau))_{x}( \pm 2 L)\right|
$$

for all $(x, s) \in[-2 L, 2 L] \times[0, t-\tau)$. The continuity of $z(x, s)$ implies that

$$
\begin{aligned}
\| z(t & -\tau) \|_{\infty,[-2 L, 2 L]} \leq \sup _{0 \leq s^{\prime} \leq t-\tau}\left|K\left(s^{\prime}\right) * f(u(\tau))_{x}( \pm 2 L)\right| \\
& \leq \sup _{0 \leq s^{\prime} \leq t-\tau} W_{+}^{L}\left(f(u(\tau))_{x}, 0, s^{\prime}\right) \\
& \leq \sup _{0 \leq s^{\prime} \leq t-\tau}\left(\left\|f(u(\tau))_{x}\right\|_{\infty,( \pm \infty, \pm L]}+\left\|f(u(\tau))_{x}\right\|_{\infty} \exp \left(-\frac{L^{2}}{4 \mu s^{\prime}}\right)\right) \\
& =\left\|f(u(\tau))_{x}\right\|_{\infty,( \pm \infty, \pm L]}+\left\|f(u(\tau))_{x}\right\|_{\infty} \exp \left(-\frac{L^{2}}{4 \mu(t-\tau)}\right) .
\end{aligned}
$$

Thus,

$$
\begin{aligned}
& \int_{0}^{t}\|z(t-s)\|_{\infty,[-2 L, 2 L]} d s \\
& \quad \leq \int_{0}^{t} M\left\|u_{x}(s)\right\|_{\infty,( \pm \infty, \pm L]} d s+C(T)\left\|u_{0}\right\|_{\infty} \sqrt{t} \exp \left(-\frac{L^{2}}{4 \mu t}\right)
\end{aligned}
$$

by using the bound $\left\|f(u(\tau))_{x}\right\|_{\infty} \leq C(T)\left\|u_{0}\right\|_{\infty} / \sqrt{\tau}$.

Theorem 3.5. Let $u(x, t)$ and $u^{2 L}(x, t)$ be the solutions of the Cauchy problem (1.1)-(1.2) and the B.V.P. (1.1)-(1.3) respectively. Then

$$
\begin{aligned}
& \left\|u(t)-u^{2 L}(t)\right\|_{\infty,[-2 L, 2 L]} \\
& \begin{aligned}
\leq C(T)\left[\left\|u_{0}-u^{ \pm}\right\|_{\infty,( \pm \infty, \pm L]}+\left(\left\|u_{0}-u^{ \pm}\right\|_{\infty}\right.\right. & \left.+\left\|u_{0}\right\|_{\infty}\right) \exp \left(-\frac{L^{2}}{4 \mu t}\right) \\
& \left.\quad+\int_{0}^{t}\left\|u_{x}(s)\right\|_{\infty,( \pm \infty, \pm L]} d s\right] .
\end{aligned}
\end{aligned}
$$

Proof. For $x \in[-2 L, 2 L]$ and $t>0$,

$$
\begin{aligned}
\mid u(x, t) & -u^{2 L}(x, t) \mid \\
\leq & \left|K(t) * u_{0}(x)-\left(G^{2 L}(t) *_{B}\left(u_{0}-h^{2 L}\right)(x)+h^{2 L}(x)\right)\right| \\
& +\left|\int_{0}^{t} K(t-s) * f(u(s))_{x} d s-\int_{0}^{t} G^{2 L}(t-s) *_{B} f\left(u^{2 L}(s)\right) d s\right| .
\end{aligned}
$$


Apply Lemma 3.3 to the first term and triangulate the second term. Then the inequality becomes

$$
\begin{aligned}
\mid u(x, t) & -u^{2 L}(x, t) \mid \leq \max \left(W_{+}^{L}\left(u_{0}, u^{+}, t\right), W_{-}^{L}\left(u_{0}, u^{-}, t\right)\right) \\
& +\left|\int_{0}^{t} K(t-s) * f(u(s))_{x} d s-\int_{0}^{t} G^{2 L}(t-s) *_{B} f(u(s))_{x} d s\right| \\
& +\mid \int_{0}^{t} G^{2 L}(t-s) *_{B}\left(f(u(s))_{x} d s-\int_{0}^{t} G^{2 L}(t-s) *_{B} f\left(u^{2 L}(s)\right) d s \mid\right. \\
\leq & \max \left(W_{+}^{L}\left(u_{0}, u^{+}, t\right), W_{-}^{L}\left(u_{0}, u^{-}, t\right)\right) \\
& +\int_{0}^{t} M\left\|u_{x}(s)\right\|_{\infty},|x| \geq L+C(T)\left\|u_{0}\right\|_{\infty} \exp \left(-\frac{L^{2}}{4 \mu t}\right) \\
& +\left|\int_{0}^{t} \int_{-2 L}^{2 L} G_{y}^{2 L}(x, y, t-s)\left(f(u(y, s))-f\left(u^{2 L}(y, s)\right)\right) d y d s\right|
\end{aligned}
$$

by Lemma 3.4. So we have

$$
\begin{aligned}
\mid u(t)- & u^{2 L}(t) \|_{\infty,[-2 L, 2 L]} \\
\leq & \left\|u_{0}-u^{ \pm}\right\|_{\infty,( \pm \infty, \pm L]}+\left\|u_{0}-u^{ \pm}\right\|_{\infty} \exp \left(-\frac{L^{2}}{4 \mu t}\right) \\
& +\int_{0}^{t} M\left\|u_{x}(s)\right\|_{\infty,|x| \geq L} d s+C(T)\left\|u_{0}\right\|_{\infty} \exp \left(-\frac{L^{2}}{4 \mu t}\right) \\
& +\int_{0}^{t} \frac{c_{1}}{\sqrt{t-s}} M\left\|u(s)-u^{2 L}(s)\right\|_{\infty,[-2 L, 2 L]} d s .
\end{aligned}
$$

By Corollary 2.2 with $M_{2}=0$ and the integrability of $\left\|u_{x}(s)\right\|_{\infty}$, we then have

$$
\begin{aligned}
\left\|u(t)-u^{2 L}(t)\right\|_{\infty,[-2 L, 2 L]} & \\
\leq C(T)\left(\left\|u_{0}-u^{ \pm}\right\|_{\infty,( \pm \infty, \pm L]}+\left(\left\|u_{0}-u^{ \pm}\right\|_{\infty}\right.\right. & \left.+\left\|u_{0}\right\|_{\infty}\right) \exp \left(-\frac{L^{2}}{4 \mu t}\right) \\
& \left.\quad+\int_{0}^{+} M\left\|u_{x}(s)\right\|_{\infty,|x| \geq L} d s\right)
\end{aligned}
$$

as required.

We will estimate the bound $\int_{0}^{t}\left\|u_{x}(s)\right\|_{\infty,[L, \infty)} d s$ which we need to complete the bounds of convergence rates in Theorem 3.5.

Let us first dispose of the following technicality.

Lemma 3.6. If $u_{0} \in L^{\infty}(\mathbf{R})$ and $\lim _{L \rightarrow \infty}\left\|u_{0}-u^{+}\right\|_{\infty,[L, \infty)}=0$, then there is a function $P$ of $L$ for $L>0$ and a constant $c^{\prime}$ which does not depend on $L$, such that

(i) $P(L) \downarrow 0$ as $L \rightarrow \infty$,

(ii) $P(L) \leq c^{\prime} P(2 L)$ for all $L>0$,

(iii) $\left\|u_{0}-u^{+}\right\|_{\infty,[L, \infty)} \leq P(L)$. 
Proof. Let $M_{L}=\left\|u_{0}-u^{+}\right\|_{\infty,[L, \infty)}$. We will construct a sequence $\left\{L_{i}\right\}$ such that $L_{i} \rightarrow \infty$ as $i \rightarrow \infty$ as follows: Let $L_{0}=0$ and

$$
L_{i}=\max \left(\sup \left\{L \geq L_{i-1}:\left\|u_{0}-u^{+}\right\|_{\infty,[L, \infty)} \geq \frac{1}{2^{i}} M_{0}\right\}, 2 L_{i-1}\right) .
$$

Then for each $i, L_{i}$ is finite since $M_{L} \rightarrow 0$ as $L \rightarrow \infty$. Also

and

$$
L_{i} \geq 2 L_{i-1} \quad \forall i \geq 1, \quad L_{i} \rightarrow \infty \quad \text { as } i \rightarrow \infty,
$$

$$
M_{L} \leq \frac{1}{2^{i}} M_{0} \text { for all } L>L_{i},
$$

by the definition of $L_{i}$. Now we define $P$ by $P(L)=M_{0} / 2^{i}$ if $L \in\left(L_{i}, L_{i+1}\right]$. Then (i) and (iii) are obvious. Given any $L>0$, there is an $i$ such that $L \in$ $\left(L_{i}, L_{i+1}\right]$ and then $P(L)=M_{0} / 2^{i+2}=2 P\left(L_{i+2}\right) \leq 2 P(2 L)$ since $2 L \leq 2 L_{i+1}$ and $P$ is decreasing. Thus (ii) is proved.

We denote the function $P(L)$ by $P\left(u_{0}, u^{+}\right)(L)$ if it is induced by $u_{0}$ and $u^{+}$as in Lemma 3.6.

We shall find a precise bound for

$$
\int_{0}^{t}\|u-x(s)\|_{\infty,[L, \infty)} d s
$$

in terms of $P\left(u_{0}, u^{+}\right)(L)$ and $1 / L^{j}$. A difficulty of the estimates is that the domain of dependence for the exact solution at each point $(x, t)$ is the whole space $\mathbf{R}$ whereas we demand that the desired convergence rates are in terms of the decay rate of arbitrary nonsmooth initial data $u_{0}$ over the one-sided domain $[L, \infty)$. Therefore, the auxiliary function $P\left(u_{0}, u^{+}\right)$will play a crucial role for "smoothing" the initial data in the process of the computations. We will show how this is possible in successive steps by Lemmas 3.7 and 3.8 as well as by Theorem 3.9.

Lemma 3.7 (Forward Local Property). Assume $u_{0} \in L^{\infty}(\mathbf{R})$ and $u$ is the exact solution of the Cauchy problem (1.1)-(1.2). Let

$$
\lim _{L \rightarrow \infty}\left\|u_{0}-u^{+}\right\|_{\infty,[L, \infty)}=0
$$

and let $f$ satisfy the assumptions of Theorem 2.2. For given $t \geq 0$ and $j \geq 0$ fixed, if there is a constant $c(t, j)$ which is independent of $L$ such that

$$
\int_{0}^{t}\left\|u_{x}(s)\right\|_{\infty,[L, \infty)} d s \leq c(t, j)\left(P\left(u_{0}, u^{+}\right)(L)+\frac{1}{L^{j}} M_{0}\right),
$$

then there is a $T=T\left(t, u_{0}\right)>0$ such that if $t<s \leq t+T$, then we have

$$
\begin{aligned}
& \int_{t}^{s}\left\|u_{x}(\rho)\right\|_{\infty,[L, \infty)} d \rho \\
& \quad \leq(s-t)^{1 / 4}(1+c(t, j))\left(P\left(u_{0}, u^{+}\right)(L)+\frac{1}{L^{j}} M_{0}\right),
\end{aligned}
$$

where $M_{0}=\left\|u_{0}-u^{+}\right\|_{\infty}$ and $P\left(u_{0}, u^{+}\right)$is as in Lemma 3.6. 
Lemma 3.8 (BACKWARd Local Property). Assume $u_{0}, u, f$ are as in Lemma 3.7. For given $t>0$ and $j \geq 1$, if for all $s \in(0, t)$, there is a constant $c(s, j)$ which is independent of $L$ for all $s \in(0, t)$ satisfying for all $L>0$,

$$
\begin{aligned}
& \int_{0}^{s}\left\|u_{x}(\rho)\right\|_{\infty,[L, \infty)} d \rho \\
& \quad \leq(t-s)^{1 / 4}(1+c(s, j))\left(P\left(u_{0}, u^{+}\right)(L)+\frac{1}{L^{j}} M_{0}\right) .
\end{aligned}
$$

then there is $T=T\left(t, u_{0}\right)$ such that if $0<t-T<s<t$, then we have

$$
\begin{aligned}
& \int_{s}^{t}\left\|u_{x}(\rho)\right\|_{\infty,[L, \infty)} d \rho \\
& \quad \leq(t-s)^{1 / 4}(1+c(s, j))\left(P\left(u_{0}, u^{+}\right)(L)+\frac{1}{L^{j}} M_{0}\right),
\end{aligned}
$$

where $M_{0}=\left\|u_{0}-u^{+}\right\|_{\infty}$ and $P\left(u_{0}, u^{+}\right)$is as in Lemma 3.6.

Theorem 3.9 (Global Property). Assume $u(t), u_{0}, u^{+}, f$ are as in Lemma 3.7. Then given $t>0$ and $j \geq 1$, there is a constant $c=c\left(j, t, u_{0}\right)>0$ which increases with $t$ such that, $\forall L>0$,

$$
\int_{0}^{t}\left\|u_{x}(s)\right\|_{\infty,[L, \infty)} d s \leq c\left(P\left(u_{0}, u^{+}\right)(L)+\frac{1}{L^{j}}\left\|u_{0}-u^{+}\right\|_{\infty}\right) .
$$

In a similar manner, we have the same result as in Theorem 3.9 for the bound $\int_{0}^{t}\left\|u_{x}(s)\right\|_{\infty,(-\infty,-L]} d s$ in terms of $P\left(u_{0}, u^{-}\right)(L)$ and $1 / L^{j}$. Hence our main Theorem 3.5 can be restated as follows.

Theorem 3.10. Assume

$$
\lim _{L \rightarrow \infty}\left\|u_{0}-u^{+}\right\|_{\infty,[L, \infty)}+\left\|u_{0}-u^{-}\right\|_{\infty,(-\infty, L]}=0 .
$$

Then

$$
\begin{aligned}
& \left\|u(t)-u^{2 L}(t)\right\|_{\infty,[-2 L, 2 L]} \\
& \leq c(j, t)\left(P\left(u_{0} \cdot u^{ \pm}\right)(L)+P\left(u_{0}, u^{-}\right)(-L)\right. \\
& \left.\quad+\frac{1}{L^{j}}\left\|u_{0}-u^{+}\right\|_{\infty}+\left\|u_{0}\right\|_{\infty} \exp \left(1-\frac{L^{2}}{4 \mu t}\right)\right),
\end{aligned}
$$

where $c(j, t)$ increases with $t$ and depends on $\min \left(\mu_{1}, \ldots \mu_{m}\right)$ and $M$.

Remark. (1) If $\left\|u_{0}-u^{+}\right\|_{\infty,\left[L_{1}, \infty\right)}=0$ for some $L_{1} \geq 0$ then one can take $P\left(u_{0}, u^{+}\right)(L)=c(j) L^{j}$ with $c(j)=L_{1}^{j}\left\|u_{0}-u^{+}\right\|_{\infty}$.

(2) If $\left\|u_{0}-u^{+}\right\|_{\infty,[L, \infty)} \leq c_{1} \exp \left(-c_{2} L\right)$ then one can take $P\left(u_{0}, u^{+}\right)=$ $c(j) / L^{j}$ where $c(j)$ depends on $c_{1}$ and $c_{2}$. 
Note that the function $P\left(u_{0}, u^{+}\right)(L)$ has a polynomial-like decay rate as $L \rightarrow \infty$. By the method of numerical analysis for the isentropic gas flow in Lagrangian coordinate ( $p$-system), as we can see from the graphs, although initial data decays exponentially, the solution does not decay exponentially. Thus, we believe $P\left(u_{0}, u^{ \pm}\right)$gives a plausible convergence for our work.

Proof of Lemma 3.7. The assumption that $S^{\prime}$ is invariant for (1.1)-(1.2) implies that $u(x, t) \in S^{\prime}$. Hence if $B_{2\|u(t)\|_{\infty}} \not \subset S$, then let

$$
\varepsilon=\operatorname{dist}\left(\partial S^{\prime}, \partial S \cap B_{2\|u(t)\|_{\infty}}\right)>0,
$$

otherwise let $\varepsilon=0$. For the given $j$, let

$$
\begin{aligned}
& \mathscr{A}_{t}=\left\{w:[t, t+T] \rightarrow L^{\infty} \mid \text { for all } s \in(t, t+T], w\right. \text { satisfies } \\
& \text { (a) }\|w(s)\|_{\infty} \leq 2\|u(t)\|_{\infty}, \\
& \text { (b) }\|w(s)-K(s-t) * u(t)\|_{\infty} \leq \varepsilon, \text { if } \varepsilon>0, \\
& \text { (c) } w(s) \in W^{1, \infty} \text { and }\left\|w_{x}(s)\right\|_{\infty} \leq\left(2 c_{1} / \sqrt{s-t}\right)\left\|w(t)-u^{+}\right\|_{\infty}, \\
& \text { where }\|K(t)\|_{1} \leq c_{1} / \sqrt{t}, \\
& \text { (d) } \int_{t}^{s}\left\|w_{x}(\tau)\right\|_{\infty,[L, \infty)} d \tau \\
& \left.\quad \leq(s-t)^{1 / 4}\left[(1+c(j, t)) P\left(u_{0}, u^{+}\right)(L)+M_{0} / L^{j}\right]\right\} .
\end{aligned}
$$

Define a norm $\|\cdot\|_{\mathscr{A}_{T}}$ on $\mathscr{A}_{T}$ by

$$
\|w\|_{\mathscr{A}_{T}}=\sup _{0 \leq \tau \leq T}\|w(t+\tau)\|_{\infty}+\sup _{0 \leq \tau \leq T}\left\|w_{x}(t+\tau)\right\|_{\infty} \sqrt{\tau} .
$$

It is easy to show $\left(\mathscr{A}_{T},\|\cdot\|_{\mathscr{A}_{T}}\right)$ is a Banach space. We define an operator $\mathscr{L}$ on $\mathscr{A}_{T}$ as follows:

$$
\mathscr{L}(w)=K(s-t) * u(t)-\int_{0}^{s-t} K_{x}(s-t-\tau) * f(w(t+\tau)) d \tau .
$$

We will show that, if $T$ is sufficiently small, then $\mathscr{L}$ maps $\mathscr{A}_{T}$ into itself and is contractive. Using the bound (2.5), one can prove easily that $\mathscr{L}(w)$ satisfies (a), (b), and (c) in the definition of $\mathscr{A}_{T}$ if $w \in \mathscr{A}_{T}$ and $T$ is sufficiently small.

To prove (d), we replace $u(t)$ in the expression for $\mathscr{L}(w)$ by (1.8) to obtain

$$
\begin{aligned}
\mathscr{L}(w)(s)= & K(s) * u_{0}-\int_{0}^{t} K(s-\tau) * f(u(\tau))_{x} d \tau \\
& -\int_{t}^{s} K(s-\tau) * f(w(\tau))_{x} d \tau \\
= & K(s) * u_{0}-\int_{0}^{s} K(s-\tau) * f(v(\tau))_{x} d \tau,
\end{aligned}
$$

where

$$
v(\tau)= \begin{cases}u(\tau) & \text { if } \tau \in(0, t] \\ w(\tau) & \text { if } \tau \in(t, t+T]\end{cases}
$$




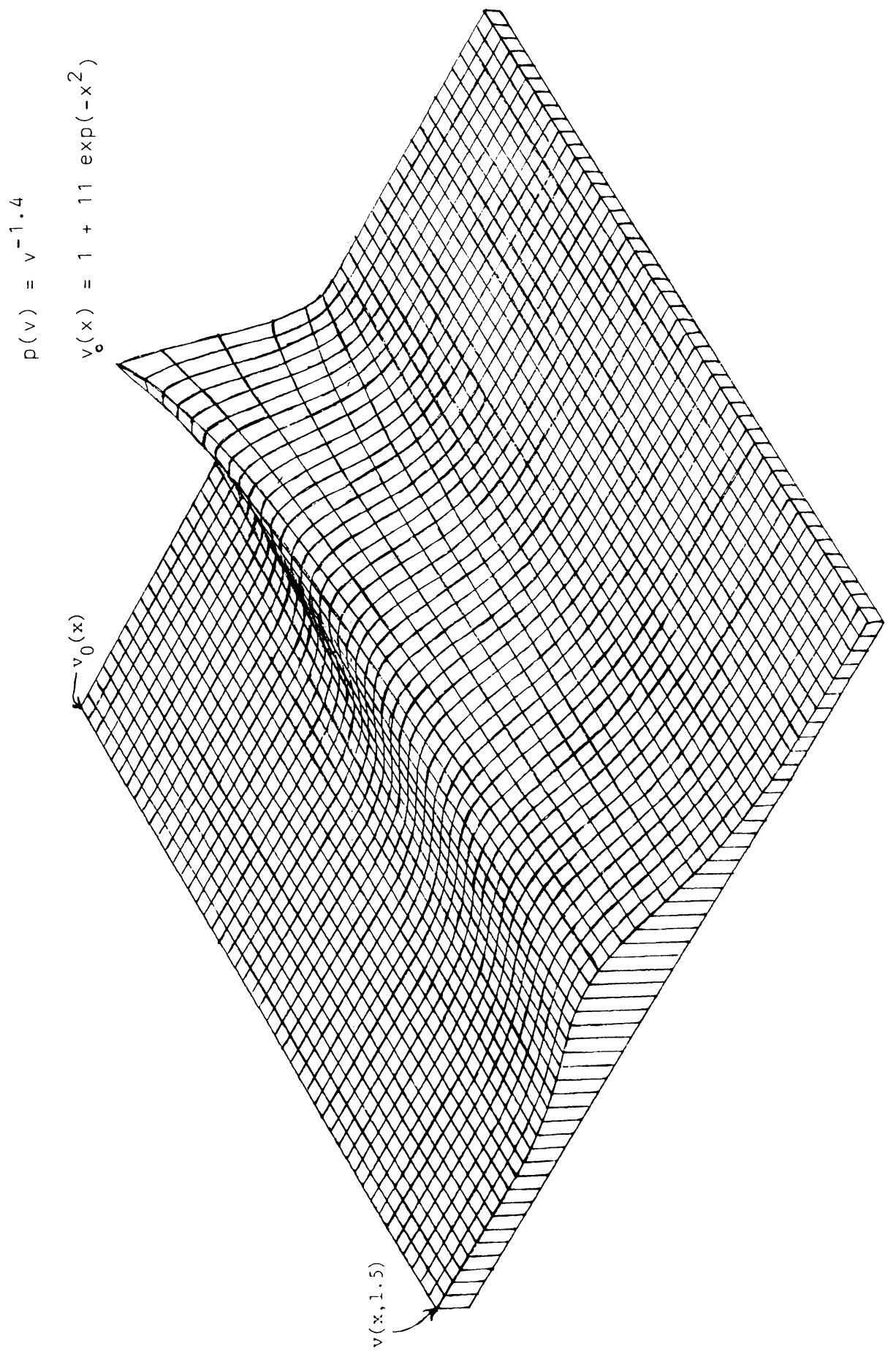




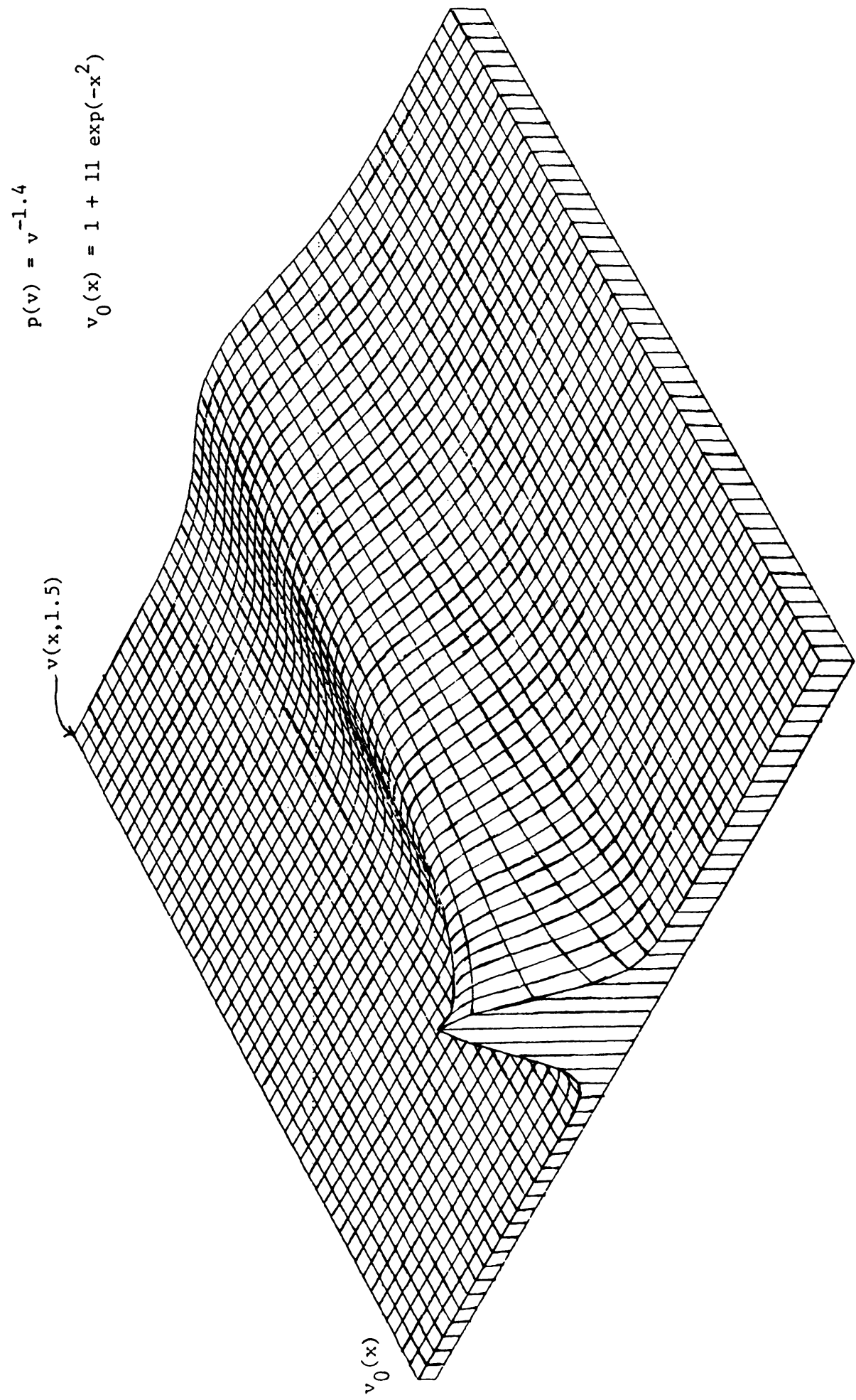




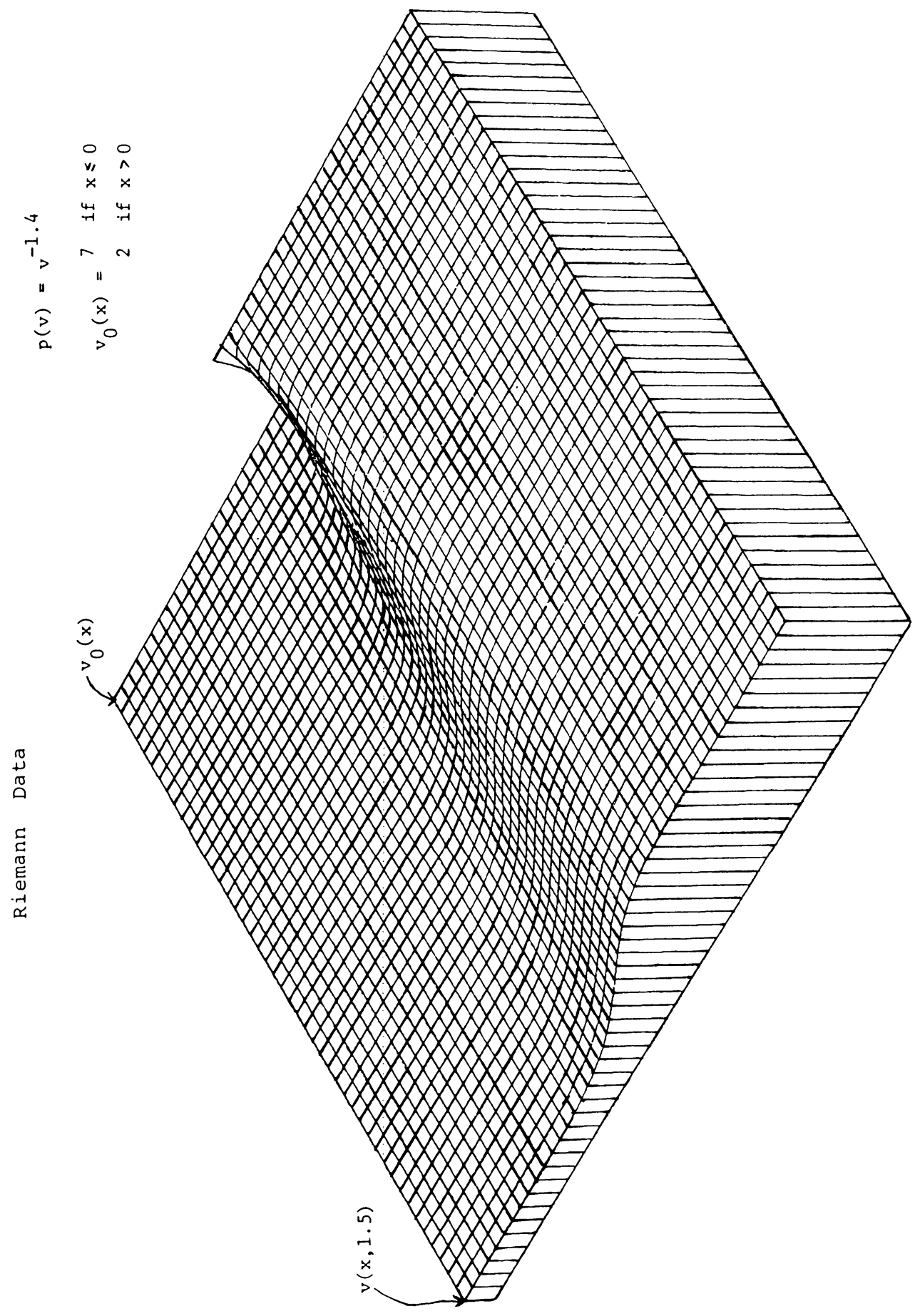


Then

$$
\begin{aligned}
\int_{t}^{s}\left\|\mathscr{L}(w)_{x}(\tau)\right\|_{\infty,[L, \infty)} d \tau \leq & \int_{t}^{s}\left\|K_{x}(\tau) *\left(u_{0}-u^{+}\right)\right\|_{\infty,[L, \infty)} d \tau \\
& +\int_{t}^{s} \int_{0}^{\tau}\left\|K_{x}(\tau-\rho) * f(v(\rho))_{x}\right\|_{\infty,[L, \infty)} d \rho \\
= & (\mathrm{A})+(\mathrm{B}) .
\end{aligned}
$$

We shall show that for small $T$ and $t<s \leq t+T$,

$$
(\mathrm{A}) \leq(s-t)^{1 / 4} P\left(u_{0}, u^{+}\right)(L)+\frac{1}{2}(s-t)^{1 / 4} \frac{M_{0}}{L^{j}}
$$

and

$$
\text { (B) } \leq(s-t)^{1 / 4}\left[c(j, t) P\left(u_{0}, u^{+}\right)(L)+c(j, t) \frac{M_{0}}{L^{j}}+\frac{1}{2} \frac{1}{L^{j}} M_{0}\right] .
$$

Thus (3.5) will be proved by (3.10) and (3.11).

We prove (3.10) as follows. By Lemma 3.2

$$
\begin{aligned}
(\mathrm{A}) & \leq \int_{t}^{s} Z_{t}^{L / 2}\left(u_{0}-u^{+}, 0, \tau\right) d \tau \\
& \leq \int_{t}^{s} \frac{C_{1}}{\sqrt{t}}\left\|u_{0}-u^{+}\right\|_{\infty,[L / 2, \infty)} d \tau+\frac{1}{2} \int_{t}^{s} \frac{\left\|u_{0}-u^{+}\right\|_{\infty}}{\sqrt{\pi \mu^{\prime}}} \exp \left(-\frac{(L / 2)^{2}}{4 \mu \tau}\right) d \tau \\
& \leq 2 c_{1}(\sqrt{s}-\sqrt{t}) P\left(u_{0}, u^{+}\right)\left(\frac{L}{2}\right)+\frac{M_{0}}{\sqrt{\pi \mu}}(\sqrt{s}-\sqrt{t}) \exp \left(-\frac{L^{2}}{16 \mu s}\right) \\
& \leq 2 c_{1}(\sqrt{s}-\sqrt{t}) \cdot c^{\prime} P\left(u_{0}, u^{+}\right)(L)+\frac{1}{\sqrt{\pi \mu}} M_{0}(\sqrt{s}-\sqrt{t}) \frac{1}{L^{j}} c(j)(16 \mu s)^{j / 2} \\
& \leq(s-t)^{1 / 4} P\left(u_{0}, u^{+}\right)(L)+\frac{1}{2}(s-t)^{1 / 4} \frac{1}{L^{j}} M_{0}
\end{aligned}
$$

for small $T$, since $(s-t)^{1 / 4} /(\sqrt{s}-\sqrt{t}) \rightarrow \infty$ as $s \rightarrow t$.

To prove (3.11), we use Lemma 3.2.

$$
\begin{aligned}
(\mathrm{B}) \leq & \int_{t}^{s} \int_{0}^{\tau} Z_{t}^{L / 2}\left(f(v(\rho))_{x}, 0, \tau-\rho\right) d \rho d \tau \\
= & \int_{\tau}^{s} \int_{0}^{\tau} \frac{c_{1}}{\sqrt{\tau-\rho}}\|f(v(\rho))\|_{\infty,[L / 2, \infty)} d \rho d \tau \\
& +\frac{1}{2} \int_{t}^{s} \int_{0}^{\tau} \frac{1}{\sqrt{\mu^{\prime} \pi(\tau-\rho)}}\left\|f(v(\rho))_{x}\right\|_{\infty} \cdot \exp \left(\frac{-(L / 2)^{2}}{4 \mu(\tau-\rho)}\right) d \rho d \tau \\
= & \left(\mathrm{B}_{1}\right)+\left(\mathrm{B}_{2}\right) .
\end{aligned}
$$

We shall show that for small $\varepsilon$ and $t<s \leq t+\varepsilon$,

$$
\left(\mathrm{B}_{1}\right) \leq(s-t) c(j, t) P\left(u_{0}, u^{+}\right)(L)+(s-t)^{1 / 4} c(j, t) \frac{1}{L^{j}} M_{0}
$$


and

$$
\left(\mathrm{B}_{2}\right) \leq \frac{1}{2}(s-t)^{1 / 4} \frac{1}{L^{j}} M_{0}
$$

Thus, (3.11) will be proved by (3.12) and (3.13). We prove (3.12) as follows:

$$
\begin{aligned}
\left(\mathrm{B}_{1}\right)= & \int_{t}^{s} \int_{0}^{t} \frac{c_{1}}{\sqrt{\tau-\rho}} M\left\|u_{x}(\rho)\right\|_{\infty,[L / 2, \infty)} d \rho d \tau \\
& +\int_{t}^{s} \int_{t}^{\tau} \frac{c_{1}}{\sqrt{\tau-\rho}} M\left\|w_{x}(\rho)\right\|_{\infty,[L / 2, \infty)} d \rho d \tau \\
= & 2 c_{1} M \int_{0}^{t}(\sqrt{s-\rho}-\sqrt{t-\rho})\left\|u_{x}(\rho)\right\|_{\infty,[L / 2, \infty)} d \rho \\
& +2 c_{1} M \int_{t}^{s} \sqrt{s-\rho}\left\|w_{x}(\rho)\right\|_{\infty,[L / 2, \infty)} d \rho
\end{aligned}
$$

(by Tonelli's Theorem for both terms)

$$
\begin{aligned}
\leq & 2 c_{1} M(s-t)^{1 / 2} \int_{0}^{t}\left\|u_{x}(\rho)\right\|_{\infty,[L / 2, \infty)} d \rho \\
& +2 c_{1} M(s-t)^{1 / 2} \int_{t}^{s}\left\|w_{x}(\rho)\right\|_{\infty,[L / 2, \infty)} d \rho \\
\leq & 2 c_{1} M(s-t)^{1 / 2}\left(c(t, j)\left(P\left(u_{0}, u^{+}\right)\left(\frac{L}{2}\right)+\frac{2^{j} M_{0}}{L^{2}}\right)\right) \quad(\text { by }(3.4)) \\
& +2 c_{1} M(s-t)^{1 / 2}\left((s-t)^{1 / 4}(1+c(t, j))\left(P\left(u_{0}, u^{+}\right)\left(\frac{L}{2}\right)+\frac{2^{j} M_{0}}{L^{2}}\right)\right) \\
\leq & (s-t)^{1 / 4} c(t, j) P\left(u_{0}, u^{+}\right)(L)+(s-t)^{1 / 4} c(t, j) \frac{M_{0}}{L^{j}} \quad\left(\text { by (d) of } \mathscr{A}_{T}\right)
\end{aligned}
$$

for small $T$, since $P\left(u_{0}, u^{+}\right)(L / 2) \leq C^{\prime} P\left(u_{0}, u^{+}\right)(L)$. We prove (3.13) as follows:

$$
\begin{aligned}
\left(\mathrm{B}_{2}\right) \leq & \int_{t}^{s} \int_{0}^{t} \frac{M}{2} \frac{1}{\sqrt{\pi \mu^{\prime}(\tau-\rho)}}\left\|u_{x}(\rho)\right\|_{\infty} \exp \left(-\frac{(L / 2)^{2}}{4 \mu(\tau-\rho)}\right) d \rho d \tau \\
& +\int_{t}^{s} \int_{t}^{\tau} \frac{M}{2 \sqrt{\pi \mu^{\prime}(\tau-\rho)}}\left\|w_{x}(\rho)\right\|_{\infty} \exp \left(-\frac{(L / 2)^{2}}{4 \mu(\tau-\rho)}\right) d \rho d \tau
\end{aligned}
$$

By

$$
\left\|u_{x}(\rho)\right\|_{\infty} \leq C\left\|u_{0}-u^{+}\right\|_{\infty} / \sqrt{\rho} \text { for } 0<\rho \leq t
$$


and by (c) in the definition of $\mathscr{A}_{T},\left(\mathrm{~B}_{2}\right)$ is bounded by

$$
\begin{aligned}
c \| u_{0}- & u^{+} \|_{\infty} \int_{t}^{s} \pi \exp \left(-\frac{L^{2}}{16 \mu \tau}\right) d \tau \\
& +c\left\|u(t)-u^{+}\right\|_{\infty} \int_{t}^{s} \pi \exp \left(-\frac{L^{2}}{16 \mu \tau}\right) d \tau \quad(\text { by }(2.5)) \\
\leq & c \cdot(s-t) \exp \left(-\frac{L^{2}}{16 \mu s}\right)\left(\left\|u_{0}-u^{+}\right\|_{\infty}+\left\|u(t)-u^{+}\right\|_{\infty}\right) \\
\leq & c(t)(s-t) \exp \left(-\frac{L^{2}}{16 \mu s}\right)\left\|u_{0}-u^{+}\right\|-\infty
\end{aligned}
$$

since

$$
\left\|u(t)-u^{+}\right\|_{\infty} \leq c(t)\left\|u_{0}-u^{+}\right\|_{\infty}
$$

where $c(t)$ is bounded on $[0, t]$. Then, by

$$
e^{-x} \leq c(j) x^{-j / 2} \quad \forall x>0
$$

we have

$$
\left(\mathrm{B}_{2}\right) \leq c(t, j) s^{j / s}(s-t)\left\|u_{0}-u^{+}\right\|_{\infty} \frac{1}{L^{j}} \leq \frac{1}{2}(s-t)^{1 / 4} M_{0} \frac{1}{L^{j}}
$$

for small $T$ and $t<s \leq t+T$, as required. Consequently, we have shown that $\mathscr{L}$ maps $\mathscr{A}_{T}$ into itself. The fact that $\mathscr{L}$ is a contraction is shown below.

If $w, v \in \mathscr{T}$ then $w(x, t), v(x, t) \in S$ for $t \in[0, T]$ (refer to the proof of Theorem 2.3) and

$$
\begin{aligned}
\|\mathscr{L}(w)-\mathscr{L}(v)\|_{\mathscr{A}_{T}} \\
\leq \sup _{0 \leq s-t \leq T} \| \int_{0}^{s-t} K_{x}(s-t-\tau) *\left(f(w(t+\tau)-f(v(t+\tau))) d \tau \|_{\infty}\right. \\
\quad+\sup _{0 \leq s-t \leq T} \| \int_{0}^{s-t} K_{x}(s-t-\tau) *\left(f(w(t+\tau))_{x}-f(v(t+\tau))_{x} d \tau \|_{\infty}\right. \\
=(\mathrm{I})+(\mathrm{II}) .
\end{aligned}
$$

It is easy to show that

$$
\text { (I) } \leq \frac{1}{4} \sup _{0 \leq s \leq T}\|w(t+\tau)-v(t+\tau)\|_{\infty} \text { for small } T \text {. }
$$


We triangulate the term (II),

$$
\begin{aligned}
(\mathrm{II}) & \leq \sup _{0<s-t \leq T} \sqrt{s-t}\left\|\int_{0}^{s-t} K_{x}(s-t-\tau) *(D f(w(t+\tau)))\left(w_{x}(t+\tau)-v_{x}(t+\tau)\right) d \tau\right\|_{\infty} \\
& +\sup _{0<s-t \leq T} \sqrt{s-t}\left\|\int_{0}^{s-t} K_{x}(s-t-\tau) *(D f(w(t+\tau))-D f(v(t+\tau))) v_{x}(t+\tau) d \tau\right\|_{\infty} \\
\leq & \sup _{0<s-t \leq T} \sqrt{s-t} \int_{0}^{s-t} \frac{c(t+T)}{\sqrt{s-t-\tau}}\left\|w_{x}(t+\tau)-v_{x}(t+\tau)\right\|_{\infty} d \tau \\
& +\sup _{0<s-t \leq T} \sqrt{s-t} \int_{0}^{s-t} \frac{c(t+T)}{\sqrt{s-t-\tau}}\left\|w_{x}(t+\tau)-v(t+\tau)\right\|_{\infty}\left\|v_{x}(t+\tau)\right\|_{\infty} d \tau \\
\leq & \sup _{0<s-t \leq T} \sqrt{s-t} \int_{0}^{s-t} \frac{c(t+T)}{\sqrt{s-t-\tau}} \frac{1}{\sqrt{\tau}} \sup _{0<\rho \leq T}\left(\sqrt{\rho}\left\|w_{x}(t+\rho)-v_{x}(t+\rho)\right\|_{\infty}\right) d \rho \\
& +\sup _{0<s-t \leq T} \sqrt{s-t} \int_{0}^{s-t} \frac{c(t+T)}{\sqrt{s-t-\tau}}\left(\sup _{0 \leq \tau \leq T}\|w(t+\tau)-v(t+\tau)\|_{\infty}\right) \cdot \frac{\left\|v(t)-u^{+}\right\|_{\infty}}{\sqrt{\tau}} d \tau \\
\leq & \sup _{0<\tau \leq T}\left(\sqrt{\tau}\left\|w_{x}(t+\tau)-v_{x}(t+\tau)\right\|\right) c(t+T) \sqrt{T} \int_{0}^{s-t} \frac{1}{\sqrt{s-t-\tau}} \frac{1}{\sqrt{\tau}} d \tau \\
& +\left(\sup _{0 \leq \tau \leq T}\|w(t+\tau)-v(t+\tau)\|_{\infty}\right) \\
\leq & \frac{1}{4} \sup _{0 \leq \tau \leq T}\|w(t+T) \sqrt{T}\| v(t)-u^{+} \|_{\infty} \\
& (t+\tau)-v(t+\tau)\left\|_{\infty}+\frac{1}{4} \sup _{0<\tau \leq T} \sqrt{\tau}\right\| w_{x}(t+\tau)-v_{x}(t+\tau) \|_{\infty}
\end{aligned}
$$

for small $T$. As a result, we have

$$
\|\mathscr{L}(w)-L(v)\|_{\mathscr{A}_{\tau}} \leq \frac{1}{2}\|w-v\|_{\mathscr{G}} .
$$

Hence there is a $w_{0} \in \mathscr{A}_{T}$ such that $w_{0}=\mathscr{L}\left(w_{0}\right)$. By the fact that $u$ is the unique global solution of $u=\mathscr{L}(u)$, we have $w_{0}(\rho) \equiv u(\rho)$ for all $\rho \in$ $[t, t+T]$. This gives us (3.5).

Proof of Lemma 3.8. The result of Lemma 3.8 can be obtained by the same proof as that of Lemma 3.7, replacing $\mathscr{A}_{T}$ and $\mathscr{L}$ by

$$
\begin{aligned}
& \mathscr{A}_{T}=\left\{w:[t-T>0, t] \rightarrow L^{\infty} \mid \text { for all } s \in(t-T, t], w\right. \text { satisfies } \\
& \text { (a) }\|w(s)\|_{\infty} \leq 2\|u(t-T)\|_{\infty}, \\
& \text { (b) }\|w(s)-K(t-s) * u(t-T)\|_{\infty} \leq \varepsilon \text { if } \varepsilon>0, \\
& \text { (c) } w(s) \in W^{1, \infty} \text { and }\left\|w_{x}(s)\right\|_{\infty} \leq 2 c_{1}\left\|u(t)-u^{+}\right\|_{\infty} / \sqrt{t-s}, \\
& \text { (d) } \int_{s}^{t}\left\|w_{x}(\tau)\right\|_{\infty,[L, \infty)} d \tau \\
& \left.\leq(t-s)^{1 / 4}(1+c(s, j))\left[P\left(u_{0}, u^{+}\right)(L)+M_{0} / L^{j}\right]\right\}
\end{aligned}
$$

and

$$
\begin{aligned}
\mathscr{L}(w)(s)= & K(s-(t-T)) * u(t-T) \\
& -\int_{0}^{s-(t-T)} K(s-(t-T)-\tau) * f(w(t-T+\rho))_{x} d \tau .
\end{aligned}
$$


Proof of Theorem 3.9. For a fixed $j$, let

$$
\begin{aligned}
\mathscr{A}=\{ & t \geq 0 \mid \text { for all } s \in[0, t], \text { there is a constant } c(s, j) \\
& \text { which is independent of } L \text { such that } \\
& \int_{0}^{s}\left\|u_{x}(\tau)\right\|_{\infty,[L, \infty)} d \tau \leq c(s, j)\left(P\left(u_{0}, u^{+}\right)(L)+\frac{M_{0}}{L^{j}}\right),
\end{aligned}
$$

for all $L\}$.

We shall prove that $\mathscr{A}$ is open and closed so that $\mathscr{A}=R^{+} \cup\{0\}$, since $\mathscr{A}$ is not empty by Lemma 3.7 (Forward Local Property). If $t \in \mathscr{A}$ then by Lemma 3.7, there is $T_{0}=T_{0}(t)>0$ such that for all $s, 0<t \leq s \leq t+T_{0}$, we have

$$
\int_{t}^{s}\left\|u_{x}(t)\right\|_{\infty,[L, \infty)} d \tau \leq(s-t)^{1 / 4}(1+c(t, j))\left[P\left(u_{0}, u^{+}\right)(L)+\frac{M_{0}}{L^{j}}\right] .
$$

Hence

$$
\begin{aligned}
& \int_{0}^{s}\left\|u_{x}(\tau)\right\|_{\infty,[L, \infty)} d \tau \\
&=\int_{0}^{t}\left\|u_{x}(\tau)\right\|_{\infty,[L, \infty)} d \tau+\int_{t}^{s}\left\|u_{x}(\tau)\right\|_{\infty,[L, \infty)} d \tau \\
& \leq c(t, j)\left(P\left(u_{0}, u^{+}\right)(L)+\frac{M_{0}}{L^{j}}\right)+T_{0}^{1 / 4}(1+c(t, j)) \\
&+\left[P\left(u_{0}, u^{+}\right)+\frac{M_{0}}{L^{j}}\right] .
\end{aligned}
$$

Therefore, $s$ is in $\mathscr{A}$ for all $s \in\left(t, t+T_{0}\right)$ since $c(s, j)$ can be taken as $c(s, j)=c(t, j)+T_{0}^{1 / 4}(1+c(t, j))$, so that $\mathscr{A}$ is open. If there is a sequence $t_{n} \uparrow t$ and $\left\{t_{n}\right\} \in \mathscr{A}$, then for all $s \in(0, t), s<t_{n}$ for some $n$. Hence (3.13) holds for $s$. Lemma 3.8 (Backward Local Property) then implies that $t \in \mathscr{A}$. So $\mathscr{A}$ is closed as well.

\section{Applications}

In this section we apply the global existence result (Theorem 2.3) and convergence rates (Theorem 3.10) to a system arising in chemical chromatography as well as to the equation modelling the flow of compressible fluids and gases. (We refer the reader to [4] for a complete description and derivation of these equations.) In order to do this, it is necessary to show that there are invariant regions for the exact solutions of $(1.1)-(1.3))$ for each system. Now, precise conditions under which the system (1.1)-(1.3) admits an invariant region are given in [12]. These conditions include the requirement that the normal vector to such a region should at each point be a left eigenvector both of $f^{\prime}(u)$ and of $D$. The former condition is sufficiently stringent in our applications that we choose to make the latter condition automatic by taking $D$ to be a scalar matrix; that is, $D=\mu I$. 


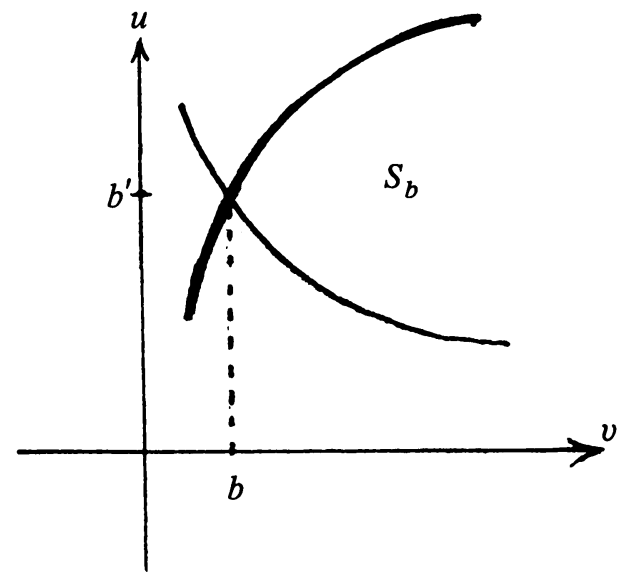

FIGURE 4.1

(a) Scalar equation.

$$
\left\{\begin{array}{l}
u_{t}+f(u)_{x}=\mu u_{x x} \\
\left.u(x, 0)=u_{0}(s) \in L^{\infty}(L, L]\right) \\
u( \pm L, t)=u^{ \pm}, \quad t>0
\end{array}\right.
$$

where $f^{\prime \prime}$ is bounded on every bounded set. By means of the maximum principle, any interval $S_{a} \subset \mathbf{R}$ is invariant for (4.a).

(b) Isentropic gas flow in Lagrangian coordinates.

$$
\begin{aligned}
& u_{t}+p(v)_{x}=\mu u_{x x}, \quad \text { on }(-L, L) \times \mathbf{R}^{+}, \\
& v_{t}-u_{x}=\mu v_{x x}
\end{aligned}
$$

where $u, v$, and $p(v)$ represent the velocity, the reciprocal of density, and pressure respectively. $p$ is a positive $C^{2}$ function and satisfies

$$
p^{\prime}<0, \quad p^{\prime \prime}>0 \text { for } v>0 .
$$

(Typically, $p(v)=c v^{-\gamma}, \gamma>1$.)

The invariant region for (4.b) (see Figure 4.1) is

$$
S_{b}=\left\{(u, v) \mid s_{b}(u, v) \leq b^{\prime} \leq r_{b}(u, v)\right\},
$$

where

$$
r_{b}(u, v)=u+\int_{b}^{r} \sqrt{-p^{\prime}(\rho)} d \rho, \quad s_{b}(u, v)=u-\int_{b}^{v} \sqrt{-p^{\prime}(\rho)} d \rho
$$

(see Smoller [12]) and the Jacobian matrix DF of $F=\left[\begin{array}{c}p(v) \\ -u\end{array}\right]$ is bounded in $S_{b}$ if $p^{\prime}$ is bounded in $S_{b}$.

(c) Isentropic gas dynamics in Eulerian coordinates.

$$
\begin{aligned}
& \rho_{t}+(\rho u)=\mu \rho_{x x}, \\
& (\rho u)_{t}+\left(\rho u^{2}+P(\rho)\right)_{x}=\mu(\rho u)_{x x},
\end{aligned} \quad \text { on }(-L, L) \times \mathbf{R}^{+},
$$


where $\rho, u$, and $P(\rho)$ are density, velocity, and pressure respectively. We assume that $P(\rho)=K \rho^{\gamma}$ where $\gamma$ is a constant satisfying $\gamma>1$ and $K$ is a positive constant. Let $w=\rho u, U=\left[\begin{array}{l}\rho \\ w\end{array}\right]$, and

$$
F(U)=\left[\begin{array}{c}
w \\
w^{2} / \rho+K \rho^{\gamma}
\end{array}\right]
$$

Then (4.c) becomes

$$
U_{t}+D F(U) U_{x}=\mu U_{x x}
$$

where

$$
D F=\left[\begin{array}{cc}
0 & \\
-(w / \rho)^{2}+K \gamma \rho^{\gamma-1} & 2 w / \rho
\end{array}\right],
$$

with left eigenvectors $l_{ \pm}=\left(-w / \rho \pm\left(K \gamma \rho^{\gamma-1}\right)^{1 / 2}, 1\right)$. Thus we can apply the result of Chueh, Conley, and Smoller [2] to (4.c) as follows: The normal vectors on the boundary curves $w^{ \pm}(\rho)$ of the invariant region are $\left(-w_{ \pm}^{\prime}(\rho), 1\right)$ which should be parallel to the left eigenvectors of $D F$. Therefore,

$$
w_{ \pm}(\rho)=\rho \text { const } \pm \rho \int_{\rho}^{c}\left(K \gamma \xi^{\gamma-3}\right)^{1 / 2} d \xi
$$

Specifically,

$$
w_{ \pm}(\rho)=\rho \frac{w_{c}}{c} \pm \int_{\rho}^{c}\left(K \gamma \xi^{\gamma-3}\right)^{1 / 2} d \xi
$$

for some positive constants $w_{c}$ and $c, w_{ \pm}$satisfy the following (i)-(iii).

(i) $w_{+}(\rho)>0$ for all $\rho \in[0, c]$;

(ii) $w_{ \pm}^{\prime}(\rho)=w_{c} / c \mp\left(K \gamma \rho^{\gamma-1}\right)^{1 / 2}+\int_{\rho}^{c}\left(K \gamma \xi^{\gamma-3}\right)^{1 / 2} d \xi$; and

(iii) $w_{ \pm}^{\prime \prime}(\rho)=\mp(\gamma-1)\left(K \gamma \rho^{\gamma-3}\right)^{1 / 2} / 2$

(so that $w_{+}^{\prime \prime}(\rho)>0$ and $w_{-}^{\prime \prime}(\rho)>0$ for all $\left.\rho>0, \gamma>1\right)$. Denote the so-called Riemann invariants by

$$
\begin{aligned}
& r_{c}(w, \rho)=\frac{\rho}{c} w_{c}+\rho \int_{\rho}^{c}\left(K \gamma \xi^{\gamma-3}\right)^{1 / 2} d \xi-w \geq 0, \\
& s_{c}(w, \rho)=\frac{\rho}{c} w_{c}-\rho \int_{\rho}^{c}\left(K \gamma \xi^{\gamma-3}\right)^{1 / 2} d \xi-w \leq 0 .
\end{aligned}
$$

Then the set

$$
\begin{aligned}
S_{c} & =\left\{(W, \rho) \mid s_{c}(w, \rho) \leq 0 \leq r_{c}(w, \rho), 0 \leq \rho \leq c\right\} \\
& =\left\{(w, \rho)|| \frac{w}{\rho}-\frac{w_{c}}{c} \mid \leq \int_{\rho}^{c}\left(K \gamma \xi^{\gamma-3}\right)^{1 / 2} d \xi\right\}
\end{aligned}
$$

is invariant for (4.c). This region can be graphed as in Figure 4.2 or Figure 4.3 in the case that $w_{c}>c \int_{\rho}^{c}\left(K \gamma \xi^{\gamma-3}\right)^{1 / 2} d \chi$ (so that $w_{-}(\rho)>0$ for all $\rho>0$ ) and in Figure 4.4 or Figure 4.5 otherwise. Obviously, DF is bounded on this invariant region $S_{c}$ for $\gamma>1$. 


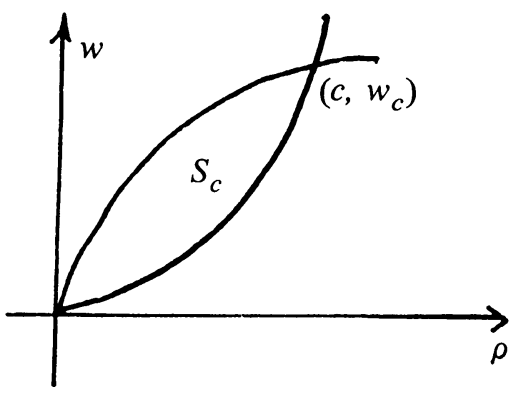

FiguRE. 4.2

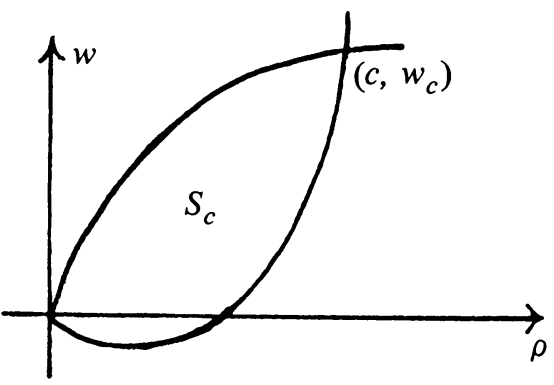

FIGURE 4.4

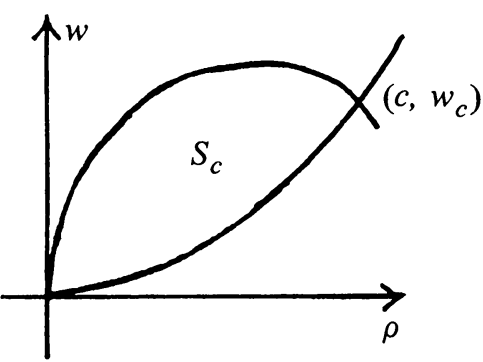

FIGURE 4.3

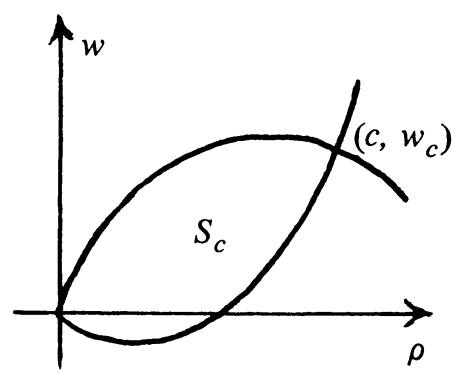

FIGURE 4.5

(d) Chemical chromatography.

$$
\frac{\partial}{\partial t}\left(\delta n_{i}+C_{i}\right)+A \frac{\partial C_{i}}{\partial x}=\mu\left(n_{i}+C_{i}\right)_{x x}, \quad i=1,2, \ldots, m .
$$

(A reference for this example is R. Aris and N. Amundson [1].) Here $n_{i}$ and $C_{i}$ are chemical concentrations which depend on $x$ and $t$, and $\delta$ and $A$ are positive constants. The system becomes closed under an additional hypothesis of the form

$$
n_{i}=\frac{N K_{i} C_{i}}{1+\sum_{j} K_{j} C_{j}},
$$

where $N$ and $K_{i}$ are positive constants. Let $u_{i}=\delta n_{i}+C_{i}$. Then by scaling out the constant $A$, (4.d) can be rewritten in the form

$$
\frac{\partial u}{\partial t}+\frac{\partial}{\partial x} C(u)=\mu u_{x x} \text { for some } \mu>0 .
$$

Applying the result of Chueh, Conley, and Smoller [2], one can show that the nonnegative octant $S_{d}$ of $u$-space is invariant for the above equation (cf. D. Hoff [8] for a detailed proof). In order to apply our theorem, it is necessary to show that $\partial C / \partial u$ is bounded in $S_{d}$. To see this, we compute the derivatives

$$
\frac{\partial u_{i}}{\partial C_{i}}=1+\frac{\delta N K_{i}\left(1+\sum_{j \neq i} K_{j} C_{j}\right)}{\left(1+\sum_{j} K_{j} C_{j}\right)^{2}}
$$


and for $k \neq i$

$$
\frac{\partial u_{i}}{\partial C_{k}}=-\frac{\delta N K_{i} K_{k} C_{i}}{\left(1+\sum_{l} K_{l} C_{l}\right)^{2}}
$$

Hence $\left|\partial u_{i} / \partial C_{k}\right|$ is bounded on $\left\{C_{l} \geq 0\right\}$ for all $i, k$. Moreover,

$$
\frac{\partial u_{i}}{\partial C_{i}}-\sum_{j \neq 1}\left|\frac{\partial u_{j}}{\partial C_{i}}\right|=1+\frac{\delta N K_{i}}{\left(1+\sum_{l} K_{l} C_{l}\right)^{2}} \geq 1
$$

Therefore, by Gershgoren's theorem, the eigenvalues of $\partial u / \partial C$ are located outside the unit circle. Therefore, $|\operatorname{det}(\partial u / \partial C)| \geq 1$, so that, by the fact that

$$
\frac{\partial C_{i}}{\partial u_{j}}=\left(\operatorname{det}\left(\frac{\partial u}{\partial C}\right)\right)^{-1} \quad\left(\text { Adjoint Matrix of } \frac{\partial u}{\partial C}\right)_{i j},
$$

$\partial C / \partial u$ is bounded in $S_{d}$.

\section{REFERENCES}

1. R. Aris and N. Amundson, Mathematical methods in chemical engineering, vol. 2, PrenticeHall, Englewood Cliffs, N. J., 1973.

2. C. N. Chueh, C. C. Conley and J. Smoller, Positively invariant regions for systems of nonlinear diffusion equations, Math. J. Indiana Univ. 26 (1977), 373-379.

3. C. Collins, Length dependence of the solutions of Fitzhugh-Nagumo equations, Trans. Amer. Math. Soc. 280 (1983).

4. R. Courant and K. O. Friedrich, Supersonic flow and shock wave, Springer-Verlag, 1948.

5. R. J. DiPerna, Convergence of the viscosity method for isentropic gas dynamics, Comm. Math. Phys. 91 (1983), 1-30.

6. J. Hale, Theory of functional differential equations, Springer-Verlag, 1976.

7. D. Hoff and J. Smoller, Error bounds for finite difference approximations for a class of nonlinear parabolic systems, Math. Comp. 45 (1985), 35-49.

8. D. Hoff, Invariant regions for systems of conservation laws, Trans. Amer. Math. Soc. 289, (1985), 591-610.

9. H. J. Kuo, Spatial truncation and finite difference schemes for systems of parabolic conservation laws, Ph.D. thesis, Indiana University, 1986.

10. O. A. Ladyzhenskaya, Linear and quasi-linear equations of parabolic type, 1968.

11. T. Nishida and J. Smoller, A class of convergent finite difference schemes for certain nonlinear parabolic systems, Comm. Pure Appl. Math. 36 (1983), 785-808.

12. J. Smoller, Shock wave and reaction-diffusion equations, Springer-Verlag, 1983.

Department of Mathematics, Indiana University, Bloomington, Indiana 47405

Current address: Department of Applied Mathematics, National Chung-Hsing University, Taichung (400), Taiwan, R.O.C. 